QUARTERLY OF APPLIED MATHEMATICS

VOLUME LXVI, NUMBER 4

DECEMBER 2008, PAGES 743-770

S 0033-569X(08)01115-9

Article electronically published on October 3, 2008

\title{
ASYMPTOTIC BEHAVIOR OF A NONISOTHERMAL GINZBURG-LANDAU MODEL
}

\author{
$\mathrm{BY}$ \\ MAURIZIO GRASSELli (Dipartimento di Matematica, Politecnico di Milano, Milano 20133, Italy), \\ HAO WU (School of Mathematical Sciences, Fudan University, Shanghai 200433, China), \\ AND \\ SONGMU ZHENG (Institute of Mathematics, Fudan University, Shanghai 200433, China)
}

Abstract. We analyze a system of nonlinear parabolic equations which describes the evolution of an order parameter $f$ and the relative temperature $v$ in a superconducting material occupying a bounded domain $\Omega \subset \mathbb{R}^{n}, n \leq 3$. Here the dependent variable $f$ is subject to the homogeneous Neumann boundary condition, while $v$ is equal to a timedependent Dirichlet datum $\tilde{u}$ on the boundary. Therefore, the corresponding dynamical system is nonautonomous. Our main goal is to analyze the asymptotic behavior of its solutions. We first show that the system has a bounded absorbing set and a global attractor which are uniform with respect to a sufficiently general class of $\tilde{u}$. Then, we give sufficient conditions on $\tilde{u}$ which ensure the convergence of a given trajectory to a single stationary state and we estimate the convergence rate. Finally, we demonstrate the existence of an exponential attractor of finite fractal dimension for quasi-periodic or stabilizing boundary data $\tilde{u}$.

1. Introduction. The present paper is concerned with the asymptotic behavior of the global solutions to a Ginzburg-Landau model which describes a superconducting material subject to thermal effects. This model was considered in the recent paper [3]. The authors extend the traditional formulation of the problem by taking the temperature as an additional dependent variable. Accordingly, together with the Gor'kov-Eliashberg

Received July 15, 2007.

2000 Mathematics Subject Classification. Primary 35K55; Secondary 35B40, 35B41, 80A22, 82D55.

Key words and phrases. Ginzburg-Landau systems, phase-field equations, nonautonomous dynamical systems, global attractors, convergence to equilibria, Łojasiewicz-Simon inequality, exponential attractors.

Dr. Songmu Zheng is the corresponding author.

E-mail address: maurizio.grasselli@polimi.it

E-mail address: haowufd@yahoo.com

E-mail address: songmuzheng@yahoo.com 
system, they introduce an evolution equation for the relative temperature (cf. the terminology used in [3]). Then they focus on the case in which only variations of the concentration of superconducting electrons and the variation of the temperature are allowed, neglecting the electromagnetic field. These assumptions led the authors to the study of the following system governing the order parameter $f$, describing the concentration of one of the two phases, and the relative temperature $v$ :

$$
\left\{\begin{array}{l}
f_{t}=\frac{1}{k^{2}} \Delta f-\left(f^{2}-1+v\right) f, \quad \text { in } \Omega \times(\tau, \infty), \\
v_{t}-f f_{t}=\eta \Delta v, \quad \text { in } \Omega \times(\tau, \infty), \\
\partial_{\nu} f=0, \quad v=\tilde{u}, \quad \text { on } \Gamma \times(\tau, \infty), \\
\left.f\right|_{t=\tau}=f_{0},\left.\quad u\right|_{t=\tau}=v_{0} \quad \text { in } \Omega .
\end{array}\right.
$$

Here, $\tau \in \mathbb{R}$ is a given initial time, $\Omega \subset \mathbb{R}^{n}, n \leq 3$, is a bounded domain with smooth boundary $\Gamma$ whose unit outward normal is denoted by $\nu, k$ is the Ginzburg-Landau parameter and $\eta>0$ is a suitable positive constant related to the conductivity of the material. The first two equations of system (1.1) can be written in a more general form as follows:

$$
\left\{\begin{array}{l}
f_{t}=\frac{1}{k^{2}} \Delta f-\phi(f)+\lambda^{\prime}(f) v, \quad \text { in } \Omega \times(\tau, \infty), \\
v_{t}+\lambda^{\prime}(f) f_{t}=\eta \Delta v, \quad \text { in } \Omega \times(\tau, \infty),
\end{array}\right.
$$

where $\phi$ and $\lambda$ are smooth functions. We recall that $\phi$ is typically the derivative of a double-well potential (e.g., $\phi(w)=w^{3}-w$ ) and $\lambda$ has a quadratic controlled growth. Notice that from the mathematical point of view the first two equations of problem (1.1) are quite similar to the well-known phase-field equations of Caginalp type (see [7]; cf. also [6, 18, 35]), though (1.1) characterizes a second order phase transition while the Caginalp model characterizes the first order phase transition, as explained in [3, 17], with the major difference that the coefficient of $f_{t}$ is no longer a constant, but a function depending on the order parameter $f$. In the case of a linear $\lambda$, the global existence result with homogeneous boundary conditions was proved in [7] under a restriction which was then removed in [15. The latter paper also contains global existence and uniqueness results with various boundary conditions, as well as the first detailed analysis of the asymptotic behavior of the solutions. Furthermore, equations (1.2) were studied as a dissipative dynamical system in [4, 5, 2, 31] and in [30, 32. In addition, it is worth recalling [40] for convergence to equilibria, and [38, 9] for singular $\phi$. All these papers deal essentially with boundary conditions of Neumann-Neumann or Neumann-Dirichlet type which can be nonhomogeneous (see, e.g., [15]), but they are always time-independent. More recently, well-posedness and asymptotic behavior of solutions for (1.2) with $\lambda$ linear and homogeneous dynamical boundary conditions have been studied in [10, 19, 20, 37]. The case of a "quadratic" $\lambda$ was first considered in [33. In particular, the author demonstrated the existence of the global attractor and constructed an exponential attractor (see [22, Secs. 8 and 9] for improvements). In [21, system (1.2) with memory effects in the heat equation and a time-dependent heat source was analyzed as a dynamical system. Convergence to single equilibria via Łojasiewicz-Simon inequality was shown in [1], and the case of a singular $\phi$ was handled in [23. Even in the quoted papers devoted to the quadratic $\lambda$, the boundary conditions are always homogeneous 
Dirichlet-Dirichlet, Neumann-Neumann, or Neumann-Dirichlet. Therefore, with respect to the above-mentioned problems which have been associated with (1.2), the present one (1.1) has two features which have been considered so far only in [3: a nonlinear coupling and a time-dependent boundary condition. As a consequence, the study of the asymptotic behavior of the solutions requires some care because we are no longer dealing with a semigroup, but with a process, and the assumptions on $\tilde{u}$ play a key role. Here we present a rather detailed analysis which begins with the construction of a dissipative process (see Sections 2 and 3) with a uniform global attractor in the sense of Chepyzhov \& Vishik 8] (see Section 4). Then, in Section 5 we show the convergence of each trajectory to a single equilibrium using an appropriate generalization of the Łojasiewicz-Simon approach, provided that $\tilde{u}$ suitably converges to some time-independent function. An "optimal" convergence rate estimate is also obtained. Finally, in the Appendix, we demonstrate the existence of an finite-dimensional exponential attractor when $\tilde{u}$ is quasiperiodic or stabilizing. In particular, in these cases we can say that the global attractor has a finite (fractal) dimension as well. We conclude by observing that it is not difficult to check that all the results proven here for (1.1) can be extended to (1.2) with $\phi$ and $\lambda$ satisfying assumptions like, for instance, the ones considered in 22], provided that they are also real analytic in Section 5.

2. Well-posedness. The nonhomogeneous boundary condition for $v$ can be handled more easily by replacing it with a new variable. Let $\hat{u}$ be the unique solution to the following Dirichlet problem:

$$
\left\{\begin{array}{lr}
-\Delta \hat{u}(\cdot, t)=0, & \text { in } \Omega, \\
\left.\hat{u}(\cdot, t)\right|_{\Gamma}=\tilde{u}(\cdot, t), & \text { on } \Gamma,
\end{array}\right.
$$

for almost any $t \in \mathbb{R}$. Then, we introduce the new variable $u=v-\hat{u}$. It turns out that problem (1.1) can be rewritten in the form

$$
\left\{\begin{array}{l}
f_{t}=\frac{1}{k^{2}} \Delta f-\left(f^{2}-1+u+\hat{u}\right) f, \quad \text { in } \Omega \times(\tau, \infty), \\
u_{t}-f f_{t}=\eta \Delta u+r, \quad \text { in } \Omega \times(\tau, \infty), \\
\partial_{\nu} f=0, \quad u=0, \quad \text { on } \Gamma \times(\tau, \infty), \\
\left.f\right|_{t=\tau}=f_{0},\left.\quad u\right|_{t=\tau}=u_{0} \quad \text { in } \Omega,
\end{array}\right.
$$

where

$$
r(x, t)=-\hat{u}_{t}(x, t), \quad u_{0}(x)=v_{0}(x)-\hat{u}(x, \tau),
$$

for almost any $x \in \Omega$ and any $t \in[\tau, \infty)$.

Let us define

$$
\mathcal{U}=L_{l o c}^{2}\left(\mathbb{R} ; H^{\frac{1}{2}}(\Gamma)\right) \cap H_{\text {loc }}^{1}\left(\mathbb{R} ; H^{-\frac{1}{2}}(\Gamma)\right),
$$

and suppose

$$
\tilde{u} \in \mathcal{U}
$$

Thus, by the elliptic regularity theory, we have

$$
\hat{u} \in L_{l o c}^{2}\left(\mathbb{R}, H^{1}(\Omega)\right) \cap H_{l o c}^{1}\left(\mathbb{R}, L^{2}(\Omega)\right) .
$$

In particular, a well-known embedding implies $\hat{u} \in C_{l o c}\left(\mathbb{R}, L^{2}(\Omega)\right)$. 
The following well-posedness result can be easily derived using, for instance, the setting introduced in [15] or a Faedo-Galerkin method based on the a priori uniform bound formally proved in Theorem 3.1 combined with the continuity estimates shown in Lemma 3.1. Therefore, we omit the details here.

Theorem 2.1. Let (2.5) hold. If $\left(f_{0}, u_{0}\right) \in H^{1}(\Omega) \times L^{2}(\Omega)$, then, for any given $\tau \in \mathbb{R}$ and any $T>\tau$, there exists a unique weak global solution $(f, u)$ to problem (2.2) such that

$$
f \in L^{2}\left([\tau, T], H^{2}(\Omega)\right) \cap C\left([\tau, T], H^{1}(\Omega)\right), \quad f_{t} \in L^{2}\left([\tau, T], L^{2}(\Omega)\right)
$$

and

$$
u \in C\left([\tau, T], L^{2}(\Omega)\right) \cap L^{2}\left([\tau, T], H_{0}^{1}(\Omega)\right), \quad u_{t} \in L^{2}\left([\tau, T], H^{-1}(\Omega)\right) .
$$

REMARK 2.1. Theorem 2.1 can be easily rephrased in terms of the original problem (1.1).

3. The dissipative process. Theorem 2.1 entails that, for any $\tilde{u} \in \mathcal{U}$, we can construct a process $U_{\tilde{u}}(t, \tau)$, defined on the phase-space $\mathcal{H}=H^{1}(\Omega) \times L^{2}(\Omega)$, by setting

$$
(f(t), u(t))=U_{\tilde{u}}(t, \tau)\left(f_{0}, u_{0}\right)
$$

for any $t \geq \tau$, where $(f(t), u(t))$ is the unique solution to problem (2.2). Observe that we can write

$$
(f(t), v(t))=U_{\tilde{u}}(t, \tau)\left(f_{0}, u_{0}\right)-(0, \hat{u}(t)) .
$$

Therefore we can always read the results for problem (2.2) in terms of the original variables.

Following the approach of [8] for nonautonomous dynamical systems (see also [25, 26]), we now consider the Banach space $\mathcal{T}$ of translation bounded functions defined by

$$
\mathcal{T}=\left\{\tilde{u} \in \mathcal{U} \mid\|\tilde{u}\|_{\mathcal{T}}^{2}:=\sup _{\tau \in \mathbb{R}} \int_{\tau}^{\tau+1}\left(\|\tilde{u}(y)\|_{H^{\frac{1}{2}(\Gamma)}}^{2}+\left\|\tilde{u}_{t}(y)\right\|_{H^{-\frac{1}{2}}(\Gamma)}^{2}\right) d y<\infty\right\},
$$

and denote by $\tilde{\mathcal{B}}$ a bounded set in $\mathcal{T}$. In the sequel we will simply indicate by $\|\cdot\|$ the standard norm in $L^{2}(\Omega)$, even for vector functions.

Let us first recall the definition of a uniform (bounded) absorbing set.

Definition 3.1. A bounded set $B_{0} \subset \mathcal{H}$ is said to be uniformly absorbing (with respect to $\tilde{u} \in \tilde{\mathcal{B}})$ if, for any bounded set $B \subset \mathcal{H}$ and any $\tau \in \mathbb{R}$, there exists $t_{0}=$ $t_{0}(B, \tau) \geq \tau$ such that

$$
\bigcup_{\tilde{u} \in \tilde{\mathcal{B}}} U_{\tilde{u}}(t, \tau) B \subset B_{0}, \quad \forall t \geq t_{0}
$$

The existence of a uniform absorbing set is ensured by the following theorem.

TheOrem 3.1. The family of processes $\left\{U_{\tilde{u}}(t, \tau), \tilde{u} \in \tilde{\mathcal{B}}\right\}$, where $\tilde{\mathcal{B}}$ is a bounded set in $\mathcal{T}$, has a uniformly absorbing set $B_{0}$. Moreover, there exists a constant $C_{0}>0$, which depends only on the size of $\tilde{\mathcal{B}}$, such that

$$
\sup _{t \geq \tau} \int_{t}^{t+1}\left(\left\|f_{t}(y)\right\|^{2}+\|u(y)\|_{H^{1}}^{2}\right) d y \leq C_{0} .
$$


Proof. We multiply the first equation of problem (2.2) by $f_{t}+\varepsilon f$ (with $\varepsilon$ being a small positive constant), multiply the second equation by $u$, and add the results together. Then, we integrate over $\Omega$ to obtain

$$
\begin{array}{r}
\frac{d}{d t}\left[\frac{1}{2}\|u\|^{2}+\frac{1}{2 k^{2}}\|\nabla f\|^{2}+\int_{\Omega}\left(\frac{1}{4} f^{4}+\frac{\varepsilon-1}{2} f^{2}\right) d x\right] \\
+\eta\|\nabla u\|^{2}+\left\|f_{t}\right\|^{2}+\frac{\varepsilon}{k^{2}}\|\nabla f\|^{2}+\varepsilon \int_{\Omega}\left(f^{4}-f^{2}\right) d x \\
=-\varepsilon \int_{\Omega} u f^{2} d x-\varepsilon \int_{\Omega} \hat{u} f^{2} d x-\int_{\Omega} \hat{u}_{t} u d x-\int_{\Omega} \hat{u} f f_{t} d x .
\end{array}
$$

We now estimate the terms on the right hand side. By the Young and Hölder inequalities, we have

$$
\begin{gathered}
\left|\varepsilon \int_{\Omega} u f^{2} d x\right| \leq \frac{\varepsilon}{4} \int_{\Omega} f^{4} d x+\varepsilon\|u\|^{2}, \\
\left|\varepsilon \int_{\Omega} \hat{u} f^{2} d x\right| \leq \frac{\varepsilon}{4} \int_{\Omega} f^{4} d x+\varepsilon\|\hat{u}\|^{2} \\
\left|\int_{\Omega} \hat{u}_{t} u d x\right| \leq\|u\|_{H^{1}}\left\|\hat{u}_{t}\right\|_{\left(H^{1}\right)^{\prime}} \leq \frac{\eta}{2}\|\nabla u\|^{2}+\frac{C}{2 \eta}\left\|\hat{u}_{t}\right\|_{\left(H^{1}\right)^{\prime}}^{2},
\end{gathered}
$$

where in (3.9), we use the Poincaré inequality for $u$.

Thanks to the well-known Sobolev embedding theorem $(n \leq 3)$, we deduce that

$$
\begin{aligned}
& \left|\int_{\Omega} \hat{u} f f_{t} d x\right| \leq\left\|f_{t}\right\|\|f\|_{L^{4}}\|\hat{u}\|_{L^{4}} \\
& \leq \frac{1}{2}\left\|f_{t}\right\|^{2}+C\|\hat{u}\|_{L^{4}}^{2}\|f\|_{L^{4}}^{2} \leq \frac{1}{2}\left\|f_{t}\right\|^{2}+C\|\hat{u}\|_{H^{1}}^{2}\|f\|_{L^{4}}^{2} .
\end{aligned}
$$

Hereafter we denote by $C$ or $C_{i}$ generic positive constants which are independent of $f$, $u$, and $t$. Let us define the functional

$$
\Phi_{1}(t)=\frac{1}{2}\|u(t)\|^{2}+\frac{1}{2 k^{2}}\|\nabla f(t)\|^{2}+\int_{\Omega}\left(\frac{1}{4} f^{4}(t)+\frac{\varepsilon-1}{2} f^{2}(t)\right) d x .
$$

Then we easily obtain

$$
C_{1}\left(\|(f, u)\|_{H^{1} \times L^{2}}^{2}+\|f\|_{L^{4}}^{4}\right)-C_{3} \leq \Phi_{1} \leq C_{2}\left(\|(f, u)\|_{H^{1} \times L^{2}}^{2}+\|f\|_{L^{4}}^{4}\right)+C_{3} .
$$

On the other hand, we have

$$
\Phi_{2}(t)=\Phi_{1}(t)+C_{3} \geq C_{1}\left(\|(f(t), u(t))\|_{H^{1} \times L^{2}}^{2}\right) \geq 0 .
$$

Thus, taking $\varepsilon$ small enough and using the Young inequality, we deduce from (3.6)-(3.12) that

$$
\frac{d}{d t} \Phi_{2}(t)+\gamma \Phi_{2}(t) \leq C_{4}\left(\|\hat{u}\|^{2}+\left\|\hat{u}_{t}\right\|_{\left(H^{1}\right)^{\prime}}^{2}\right)+C_{5}\|\hat{u}\|_{H^{1}}^{2} \sqrt{\Phi_{2}(t)}+C_{6} .
$$

Since $\tilde{\mathcal{B}}$ is a bounded set in $\mathcal{T}$, there holds

$$
\sup _{t \geq \tau} \int_{t}^{t+1}\left(\|\hat{u}(y)\|_{H^{1}}^{2}+\left\|\hat{u}_{t}(y)\right\|_{\left(H^{1}\right)^{\prime}}^{2}\right) d y<C_{7}
$$


for some $C_{7}>0$. Then it follows from [21, Lemma 2.5] that the following dissipative estimate holds:

$$
\Phi_{2}(t) \leq 2 \Phi_{2}(\tau) e^{-\gamma(t-\tau)}+Q\left(C_{7}\right), \quad t \geq \tau,
$$

for some monotone increasing positive function $Q$. This implies the existence of a uniform absorbing set. Then, integrating (3.6) with respect to time over $(t, t+1)$, we get (3.5).

Theorem 3.1 expresses the dissipativity of the process $U_{\tilde{u}}(t, \tau)$; that is, the fact that the long term dynamics concentrate within a bounded region of the phase-space, uniformly with respect to $\tilde{u} \in \tilde{\mathcal{B}}$. We conclude this section by proving the Lipschitz continuous dependence of the process on the initial data as well as on the boundary data.

Lemma 3.1. Let $\tilde{u}_{i} \in \mathcal{U},\left(f_{0 i}, u_{0 i}\right) \in B_{0}$, and $\left(f_{i}(t), u_{i}(t)\right)=U_{\tilde{u}_{i}}(t, \tau)\left(f_{0 i}, u_{0 i}\right)$, for $i=1,2$. Then, there holds

$$
\begin{aligned}
& \left\|\left(f_{1}-f_{2}\right)(t)\right\|_{H^{1}}^{2}+\left\|\left(u_{1}-u_{2}\right)(t)\right\|^{2} \\
\leq & C e^{C \beta(t)}\left[\left\|f_{10}-f_{20}\right\|_{H^{1}}^{2}+\left\|u_{10}-u_{20}\right\|^{2}\right. \\
+ & \left.\int_{\tau}^{t}\left(\left\|\left(\tilde{u}_{1}-\tilde{u}_{2}\right)(y)\right\|_{H^{\frac{1}{2}}(\Gamma)}^{2}+\left\|\left(\tilde{u}_{1 t}-\tilde{u}_{2 t}\right)(y)\right\|_{H^{-\frac{3}{2}}(\Gamma)}^{2}\right) d y\right],
\end{aligned}
$$

for all $t \geq \tau$. Here $C$ is a positive constant independent of time, and

$$
\begin{aligned}
\beta(t) & :=\int_{\tau}^{t}\left(1+\left\|\tilde{u}_{2}(y)\right\|_{H^{\frac{1}{2}(\Gamma)}}^{2}+\left\|\tilde{u}_{1}(y)\right\|_{H^{\frac{1}{2}(\Gamma)}}^{2}+\left\|u_{1}(y)\right\|_{H^{1}}^{2}+\left\|u_{2}(y)\right\|_{H^{1}}^{2}\right. \\
& \left.+\left\|f_{1}(y)\right\|_{H^{2}}^{2}+\left\|f_{2}(y)\right\|_{H^{2}}^{2}+\left\|f_{1 t}(y)\right\|^{2}+\left\|f_{2 t}(y)\right\|^{2}\right) d y .
\end{aligned}
$$

Proof. Set $f:=f_{1}-f_{2}$ and $u:=u_{1}-u_{2}$. Then we have

$$
\begin{gathered}
f_{t}=\frac{1}{k^{2}} \Delta f-\left(u_{1} f_{1}-u_{2} f_{2}\right)-\left(\hat{u}_{1} f_{1}-\hat{u}_{2} f_{2}\right)+f-f_{1}^{3}+f_{2}^{3}, \\
u_{t}-f_{1} f_{1 t}+f_{2} f_{2 t}=\eta \Delta u-\hat{u}_{1 t}+\hat{u}_{2 t} .
\end{gathered}
$$

We multiply equation (3.19) by $f_{t}+\varepsilon f$ and equation (3.20) by $u$, then integrate on $\Omega$ and add the results together to get

$$
\begin{aligned}
& \frac{d}{d t}\left[\frac{1}{2}\|u\|^{2}+\frac{1}{2 k^{2}}\|\nabla f\|^{2}+\frac{\varepsilon}{2}\|f\|^{2}\right]+\eta\|\nabla u\|^{2}+\left\|f_{t}\right\|^{2}+\frac{\varepsilon}{k^{2}}\|\nabla f\|^{2} \\
= & \varepsilon \int_{\Omega} f^{2} d x-\varepsilon \int_{\Omega}\left(u_{1} f_{1}-u_{2} f_{2}\right) f d x-\varepsilon \int_{\Omega}\left(\hat{u}_{1} f_{1}-\hat{u}_{2} f_{2}\right) f d x \\
& -\varepsilon \int_{\Omega} f^{2}\left(f_{1}^{2}+f_{1} f_{2}+f_{2}^{2}\right) d x-\int_{\Omega}\left(u_{1} f_{1}-u_{2} f_{2}\right) f_{t} d x-\int_{\Omega}\left(\hat{u}_{1} f_{1}-\hat{u}_{2} f_{2}\right) f_{t} d x \\
& +\int_{\Omega} f f_{t} d x-\int_{\Omega} f f_{t}\left(f_{1}^{2}+f_{1} f_{2}+f_{2}^{2}\right) d x \\
& -\int_{\Omega}\left(\hat{u}_{1 t}-\hat{u}_{2 t}\right) u d x+\int_{\Omega}\left(f_{1} f_{1 t}-f_{2} f_{2 t}\right) u d x .
\end{aligned}
$$


Let us estimate all the terms on the right hand side. We have

$$
\begin{aligned}
& \varepsilon\left|\int_{\Omega}\left(u_{1} f_{1}-u_{2} f_{2}\right) f d x\right|=\varepsilon\left|\int_{\Omega}\left(u_{1} f_{1}-u_{2} f_{1}\right) f d x\right|+\varepsilon\left|\int_{\Omega}\left(u_{2} f_{1}-u_{2} f_{2}\right) f d x\right| \\
& \leq \varepsilon\|u\|\left\|f_{1}\right\|_{L^{6}}\|f\|_{L^{3}}+\varepsilon\left\|u_{2}\right\|\|f\|_{L^{4}}^{2} \\
& \leq C \varepsilon\left(\|u\|^{2}+\|f\|_{H^{1}}^{2}\right) \\
& \varepsilon\left|\int_{\Omega}\left(\hat{u}_{1} f_{1}-\hat{u}_{2} f_{2}\right) f d x\right| \leq C \varepsilon\left(\|\hat{u}\|^{2}+\|f\|_{H^{1}}^{2}+\left\|\hat{u}_{2}\right\|\|f\|_{H^{1}}^{2}\right), \\
& \varepsilon\left|\int_{\Omega} f^{2}\left(f_{1}^{2}+f_{1} f_{2}+f_{2}^{2}\right) d x\right| \leq \varepsilon\|f\|_{L^{4}}^{2}\left\|f_{1}^{2}+f_{1} f_{2}+f_{2}^{2}\right\| \\
& \leq C \varepsilon\|f\|_{H^{1}}^{2}, \\
& \left|\int_{\Omega}\left(u_{1} f_{1}-u_{2} f_{2}\right) f_{t} d x\right| \leq\left|\int_{\Omega}\left(u_{1} f_{1}-u_{1} f_{2}\right) f_{t} d x\right|+\left|\int_{\Omega}\left(u_{1} f_{2}-u_{2} f_{2}\right) f_{t} d x\right| \\
& \leq\left\|u_{1}\right\|_{L^{6}}\|f\|_{L^{3}}\left\|f_{t}\right\|+\left\|f_{2}\right\|_{L^{\infty}}\|u\|\left\|f_{t}\right\| \\
& \leq \frac{1}{4}\left\|f_{t}\right\|^{2}+C\left\|u_{1}\right\|_{H^{1}}^{2}\|f\|_{H^{1}}^{2}+C\left\|f_{2}\right\|_{H^{2}}^{2}\|u\|^{2}, \\
& \left|\int_{\Omega}\left(\hat{u}_{1} f_{1}-\hat{u}_{2} f_{2}\right) f_{t} d x\right| \leq\left\|\hat{u}_{1}\right\|_{L^{6}}\|f\|_{L^{3}}\left\|f_{t}\right\|+\left\|f_{2}\right\|_{L^{6}}\|\hat{u}\|_{L^{3}}\left\|f_{t}\right\| \\
& \leq \frac{1}{4}\left\|f_{t}\right\|^{2}+C\left\|\hat{u}_{1}\right\|_{H^{1}}^{2}\|f\|_{H^{1}}^{2}+C\|\hat{u}\|_{H^{1}}^{2}, \\
& \left|\int_{\Omega} f f_{t} d x\right| \leq \frac{1}{8}\left\|f_{t}\right\|^{2}+C\|f\|^{2} \\
& \left|\int_{\Omega} f f_{t}\left(f_{1}^{2}+f_{1} f_{2}+f_{2}^{2}\right) d x\right| \leq\left\|f_{t}\right\|\|f\|_{L^{6}}\left\|f_{1}^{2}+f_{1} f_{2}+f_{2}^{2}\right\|_{L^{3}} \\
& \leq \frac{1}{8}\left\|f_{t}\right\|^{2}+C\|f\|_{H^{1}}^{2}, \\
& \left|\int_{\Omega}\left(\hat{u}_{1 t}-\hat{u}_{2 t}\right) u d x\right| \leq\left\|\hat{u}_{t}\right\|_{\left(H^{1}\right)^{\prime}}\|u\|_{H^{1}} \leq C\left\|\hat{u}_{t}\right\|_{\left(H^{1}\right)^{\prime}}^{2}+\frac{\eta}{4}\|\nabla u\|^{2}, \\
& \left|\int_{\Omega}\left(f_{1} f_{1 t}-f_{2} f_{2 t}\right) u d x\right| \\
& \leq\left|\int_{\Omega}\left(f_{1} f_{1 t}-f_{1} f_{2 t}\right) u d x\right|+\left|\int_{\Omega}\left(f_{1} f_{2 t}-f_{2} f_{2 t}\right) u d x\right| \\
& \leq\left\|f_{t}\right\|\|u\|\left\|f_{1}\right\|_{L^{\infty}}+\left\|f_{2 t}\right\|\|u\|_{L^{3}}\|f\|_{L^{6}} \\
& \leq \frac{1}{8}\left\|f_{t}\right\|^{2}+C\left\|f_{1}\right\|_{H^{2}}^{2}\|u\|^{2}+\frac{\eta}{4}\|\nabla u\|^{2}+C\|u\|^{2}+C\left\|f_{2 t}\right\|^{2}\|f\|_{H^{1}}^{2} \text {. }
\end{aligned}
$$

Putting these estimates together, we get

$$
\begin{aligned}
\frac{d}{d t} & {\left[\frac{1}{2}\|u\|^{2}+\frac{1}{2 k^{2}}\|\nabla f\|^{2}+\frac{\varepsilon}{2}\|f\|^{2}\right]+\frac{\eta}{2}\|\nabla u\|^{2}+\frac{1}{8}\left\|f_{t}\right\|^{2}+\frac{\varepsilon}{k^{2}}\|\nabla f\|^{2} } \\
& \leq m_{1}(t)\left(\|u\|^{2}+\|f\|_{H^{1}}^{2}\right)+m_{2}(t)
\end{aligned}
$$


where

$$
\begin{gathered}
m_{1}(t):=C\left(1+\left\|\hat{u}_{2}(t)\right\|^{2}+\left\|\hat{u}_{1}(t)\right\|_{H^{1}}^{2}+\left\|u_{1}(t)\right\|_{H^{1}}^{2}+\left\|f_{1}(t)\right\|_{H^{2}}^{2}+\left\|f_{2}(t)\right\|_{H^{2}}^{2}+\left\|f_{2 t}(t)\right\|^{2}\right), \\
m_{2}(t):=C\left(\|\hat{u}(t)\|_{H^{1}}^{2}+\left\|\hat{u}_{t}(t)\right\|_{\left(H^{1}\right)^{\prime}}^{2}\right) .
\end{gathered}
$$

Observe now that

$$
\begin{aligned}
\left\|\Delta f_{i}\right\| & \leq k^{2}\left(\left\|f_{i t}\right\|+\left\|f_{i}^{3}\right\|+\left\|f_{i}\right\|+\left\|f_{i}\right\|_{L^{4}}\left\|u_{i}\right\|_{L^{4}}+\left\|\hat{u}_{i}\right\|_{L^{4}}\left\|f_{i}\right\|_{L^{4}}\right) \\
& \leq C\left\|f_{i t}\right\|+C\left\|u_{i}\right\|_{H^{1}}+C\left\|\hat{u}_{i}\right\|_{H^{1}}+C .
\end{aligned}
$$

Then, it follows from (3.5) and (3.34) that

$$
\int_{t}^{t+1}\left\|f_{i}(y)\right\|_{H^{2}}^{2} d y \leq C, \quad \forall t \geq \tau
$$

Hence, we can apply a suitable Gronwall inequality (see, for instance, 21, Lemma 2.4]) to (3.31). This yields

$$
\|u(t)\|^{2}+\|f(t)\|_{H^{1}}^{2} \leq 2 e^{\int_{\tau}^{t} m_{1}(y) d y}\left(\left\|u_{0}\right\|^{2}+\left\|f_{0}\right\|_{H^{1}}^{2}\right)+2 e^{\int_{\tau}^{t} m_{1}(y) d y} \int_{\tau}^{t} m_{2}(y) d y,
$$

where $u_{0}:=u_{01}-u_{02}$ and $f_{0}:=f_{01}-f_{02}$. This entails (3.17).

4. The uniform attractor. In this section we establish the existence of a uniform attractor for problem (2.2). We always proceed along the lines of [8, Part 2].

Let us first recall the notion of translation compact function.

Definition 4.1. A function $g \in \mathcal{U}$ is said to be translation compact in $\mathcal{U}$ if the hull of $g$ defined as

$$
H(g)={\overline{\left\{g^{r}\right\}_{r \in \mathbb{R}}}}^{\mathcal{A}}
$$

is compact in $\mathcal{U}$, with respect to its local topology, where $g^{r}(\cdot)=g(\cdot+r)$ is the translation of $g$ by $r$. In particular, we have that $g \in \mathcal{T}$ and

$$
\|\tilde{g}\|_{\mathcal{T}} \leq\|g\|_{\mathcal{T}}, \quad \forall \tilde{g} \in H(g) .
$$

REMARK 4.1. Notice that the class of translation compact functions in $\mathcal{U}$ is fairly general. For instance, it contains the space $H_{l o c}^{1}\left(\mathbb{R} ; H^{\frac{1}{2}+\epsilon}(\Gamma)\right) \cap H_{l o c}^{2}\left(\mathbb{R} ; H^{-\frac{1}{2}+\epsilon}(\Gamma)\right)$, for each $\epsilon>0$. We refer to [8, Chapter $\mathrm{V}]$ and the references therein for more details.

Let $\tilde{u}^{\#} \in \mathcal{U}$ be a translation compact function. We want to study the asymptotic behavior of the family of processes $\left\{U_{\tilde{u}}(t, \tau), \tilde{u} \in H\left(\tilde{u}^{\#}\right)\right\}$. This family, thanks to Theorem 3.1, has a uniform absorbing set $\mathcal{B}_{0}$, and we set

$$
R_{0}=\sup _{\left(f_{0}, u_{0}\right) \in \mathcal{B}_{0}}\left\|\left(f_{0}, u_{0}\right)\right\|_{\mathcal{H}}
$$

Let us now prove the following compactness property.

Lemma 4.1. Let $\left(f_{0}, u_{0}\right) \in \mathcal{B}_{0}$. Then there exists a constant $K$ depending only on $R_{0}$ and $\left\|\tilde{u}^{\#}\right\|_{\mathcal{T}}$ such that

$$
\sup _{\tilde{u} \in H(\tilde{u} \#)}\left\|U_{\tilde{u}}(t, \tau)\left(f_{0}, u_{0}\right)\right\|_{H^{2} \times H_{0}^{1}} \leq K,
$$

for any given $\tau \in \mathbb{R}$ and all $t \geq \tau+1$. 
Proof. Multiplying the first equation of (2.2) by $-\Delta f_{t},-\kappa \Delta f$ respectively, multiplying the second equation of (2.2) by $u_{t}$, and integrating on $\Omega$, we have

$$
\begin{aligned}
\frac{d}{d t} \frac{1}{2 k^{2}} \| & \Delta f\left\|^{2}+\right\| \nabla f_{t} \|^{2} \\
= & -3 \int_{\Omega} f^{2} \nabla f \cdot \nabla f_{t} d x+\int_{\Omega} \nabla f \cdot \nabla f_{t} d x-\int_{\Omega} f \nabla(u+\hat{u}) \cdot \nabla f_{t} d x \\
& \quad-\int_{\Omega}(u+\hat{u}) \nabla f \cdot \nabla f_{t} d x \\
:= & I_{1}, \\
\frac{d}{d t} \frac{\kappa}{2} \| & \nabla f\left\|^{2}+\frac{\kappa}{k^{2}}\right\| \Delta f \|^{2}+3 \kappa \int_{\Omega} f^{2}|\nabla f|^{2} d x \\
= & \kappa\|\nabla f\|^{2}-\kappa \int_{\Omega}|\nabla f|^{2}(u+\hat{u}) d x-\kappa \int_{\Omega} f \nabla f \cdot \nabla(u+\hat{u}) d x \\
& :=I_{2}, \\
& \frac{d}{d t} \frac{\eta}{2}\|\nabla u\|^{2}+\left\|u_{t}\right\|^{2}=\int_{\Omega} f f_{t} u_{t} d x-\int_{\Omega} \hat{u}_{t} u_{t} d x:=I_{3} .
\end{aligned}
$$

Observe that the following inequalities hold:

$$
\begin{aligned}
& \left|3 \int_{\Omega} f^{2} \nabla f \cdot \nabla f_{t} d x\right| \leq \frac{1}{8}\left\|\nabla f_{t}\right\|^{2}+C\|\nabla f\|_{L^{6}}^{2}\|f\|_{L^{6}}^{4} \\
& \leq \frac{1}{8}\left\|\nabla f_{t}\right\|^{2}+C\|f\|_{H^{1}}^{4}\|f\|_{H^{2}}^{2}, \\
& \left|\int_{\Omega} \nabla f \cdot \nabla f_{t} d x\right| \leq \frac{1}{8}\left\|\nabla f_{t}\right\|^{2}+C\|\nabla f\|^{2}, \\
& \left|\int_{\Omega} f \nabla(u+\hat{u}) \cdot \nabla f_{t} d x\right| \leq \frac{1}{8}\left\|\nabla f_{t}\right\|^{2}+C\|f\|_{L^{\infty}}^{2}\|\nabla u\|^{2}+C\|f\|_{L^{\infty}}^{2}\|\nabla \hat{u}\|^{2} \\
& \leq \frac{1}{8}\left\|\nabla f_{t}\right\|^{2}+C\|f\|_{H^{2}}^{2}\left(\|\nabla u\|^{2}+\|\nabla \hat{u}\|^{2}\right), \\
& \left|\int_{\Omega}(u+\hat{u}) \nabla f \cdot \nabla f_{t} d x\right| \leq \frac{1}{8}\left\|\nabla f_{t}\right\|^{2}+C\|\nabla f\|_{L^{6}}^{2}\|u\|_{L^{3}}^{2}+C\|\nabla f\|_{L^{6}}^{2}\|\hat{u}\|_{L^{3}}^{2} \\
& \leq \frac{1}{8}\left\|\nabla f_{t}\right\|^{2}+C\|f\|_{H^{2}}^{2}\left(\|u\|_{H^{1}}^{2}+\|\hat{u}\|_{H^{1}}^{2}\right) .
\end{aligned}
$$

Hence we have

$$
\left|I_{1}\right| \leq \frac{1}{2}\left\|\nabla f_{t}\right\|^{2}+C\left(\|f\|_{H^{1}}^{4}+\|u\|_{H^{1}}^{2}+\|\hat{u}\|_{H^{1}}^{2}\right)\|f\|_{H^{2}}^{2}+C\|\nabla f\|^{2} .
$$


Similarly we have

$$
\begin{aligned}
\left|I_{2}\right| \leq \kappa\|\nabla f\|^{2}+\kappa\|\nabla f\|_{L^{4}}^{2}\|u+\hat{u}\|+\kappa\|f\|_{L^{\infty}}\|\nabla f\|\|\nabla(u+\hat{u})\| \\
\leq \kappa\|\nabla f\|^{2}+C \kappa\|f\|_{H^{2}}^{2}\left(\|u\|_{H^{1}}+\|\hat{u}\|_{H^{1}}\right), \\
\left|I_{3}\right| \leq \frac{1}{2}\left\|u_{t}\right\|^{2}+C\left\|f_{t}\right\|_{L^{3}}^{2}\|f\|_{L^{6}}^{2}+C\left\|\hat{u}_{t}\right\|^{2} \\
\leq \frac{1}{2}\left\|u_{t}\right\|^{2}+\frac{1}{4}\left\|\nabla f_{t}\right\|^{2}+C\left\|f_{t}\right\|^{2}\|f\|_{L^{6}}^{4}+C\left\|\hat{u}_{t}\right\|^{2} \\
\leq \frac{1}{2}\left\|u_{t}\right\|^{2}+\frac{1}{4}\left\|\nabla f_{t}\right\|^{2}+C\left\|f_{t}\right\|^{2}\|f\|_{H^{1}}^{4}+C\left\|\hat{u}_{t}\right\|^{2} .
\end{aligned}
$$

Let us now define

$$
\Psi(t)=\frac{1}{2 k^{2}}\|\Delta f\|^{2}+\frac{\kappa}{2}\|\nabla f\|^{2}+\frac{\eta}{2}\|\nabla u\|^{2}
$$

and observe that

$$
\begin{aligned}
& \frac{d}{d t} \Psi+\frac{1}{4}\left\|\nabla f_{t}\right\|^{2}+\frac{\kappa}{k^{2}}\|\Delta f\|^{2}+3 \kappa \int_{\Omega} f^{2}|\nabla f|^{2} d x+\frac{1}{2}\left\|u_{t}\right\|^{2} \\
\leq & C\left(\|f\|_{H^{1}}^{4}+\|u\|_{H^{1}}^{2}+\|\hat{u}\|_{H^{1}}^{2}+1\right)\|f\|_{H^{2}}^{2}+C\left\|f_{t}\right\|^{2}\left(\|f\|_{H^{1}}^{4}+1\right) \\
& +C\left\|\hat{u}_{t}\right\|^{2}+C\|\nabla f\|^{2},
\end{aligned}
$$

which implies that

$$
\frac{d}{d t} \Psi(t) \leq l_{1}(t) \Psi(t)+l_{2}(t)
$$

where

$$
\begin{aligned}
l_{1}(t):= & C\left(\|f(t)\|_{H^{1}}^{4}+\|u(t)\|_{H^{1}}^{2}+\|\hat{u}(t)\|_{H^{1}}^{2}+1\right), \\
l_{2}(t):= & C\left[\left(\|f(t)\|_{H^{1}}^{4}+\|u(t)\|_{H^{1}}^{2}+\|\hat{u}(t)\|_{H^{1}}^{2}+1\right)\|f(t)\|^{2}\right. \\
& \left.+\left\|f_{t}(t)\right\|^{2}\left(\|f(t)\|_{H^{1}}^{4}+1\right)+\left\|\hat{u}_{t}(t)\right\|^{2}+\|\nabla f(t)\|^{2}\right] .
\end{aligned}
$$

We now recall that, by definition,

$$
\|\tilde{u}\|_{\mathcal{T}} \leq\left\|\tilde{u}^{\#}\right\|_{\mathcal{T}}, \quad \forall \tilde{u} \in H\left(\tilde{u}^{\#}\right) .
$$

On the other hand, we also have (cf. (3.5))

$$
\sup _{t \geq \tau} \int_{t}^{t+1}\left(\|\nabla u(y)\|^{2}+\left\|f_{t}(y)\right\|^{2}\right) d y \leq C,
$$

where $C$ is a positive constant depending only on $R_{0}$ and $\left\|\tilde{u}^{\#}\right\|_{\mathcal{T}}$. Thus, it follows from Theorem 3.1 that

$$
\sup _{t \geq \tau} \int_{t}^{t+1} l_{1}(y) d y \leq C, \quad \sup _{t \geq \tau} \int_{t}^{t+1} l_{2}(y) d y \leq C .
$$

Using again (3.5), we easily deduce the integral bound

$$
\sup _{\tau \in \mathbb{R}} \sup _{t \geq \tau} \int_{t}^{t+1}\|\Delta f(y)\|^{2} d y \leq C .
$$


We are now able to apply, thanks to (4.19)-(4.21), a suitable adaptation of the uniform Gronwall Lemma (cf., e.g., [21, Lemma 2.3]) to conclude that

$$
\Psi(t+1) \leq C, \quad \forall t \geq \tau,
$$

which implies (4.2), because of (4.13).

Lemma 4.1 entails the existence of a uniformly absorbing compact set for the family $\left\{U_{\tilde{u}}(t, \tau), \tilde{u} \in H\left(\tilde{u}^{\#}\right)\right\}$. Now consider the extended semigroup

$$
S(t): \mathcal{H} \times H\left(\tilde{u}^{\#}\right) \rightarrow \mathcal{H} \times H\left(\tilde{u}^{\#}\right),
$$

defined by

$$
S(t)\left(f_{0}, u_{0}, \tilde{u}\right):=\left(U_{\tilde{u}}(t, 0)\left(f_{0}, u_{0}\right), \tilde{u}(t+\cdot)\right) .
$$

We also introduce the two canonical projectors $\Pi_{1}$ and $\Pi_{2}$ from $\mathcal{H} \times H\left(\tilde{u}^{\#}\right)$ onto $\mathcal{H}$ and $H\left(\tilde{u}^{\#}\right)$, respectively.

Therefore, by Lemma 3.1 and Lemma 4.1] it follows from [8, Thm. 5.1] that

THEOREM 4.1. If $\tilde{u}^{\#} \in \mathcal{U}$ is a translation compact function, then the extended semigroup $S(t)$ has a unique global attractor $\mathbb{A}$. Moreover,

(i) $\Pi_{1} \mathbb{A}=\mathcal{A}$ is the so-called uniform attractor of the family $\left\{U_{\tilde{u}}(t, \tau), \tilde{u} \in H\left(\tilde{u}^{\#}\right)\right\}$;

(ii) $\Pi_{2} \mathbb{A}=H\left(\tilde{u}^{\#}\right)$.

REMARK 4.2. We recall that the kernel $\mathcal{K}_{\tilde{u}}$ of the process $U_{\tilde{u}}(t, \tau)$ is the set consisting of all bounded and complete trajectories of the process itself, while $\mathcal{K}_{\tilde{u}}(s)$ is the kernel section at time $s, s \in \mathbb{R}$. Then, we have the following characterizations:

$$
\mathbb{A}=\bigcup_{\tilde{u} \in H(\tilde{u} \#)} \mathcal{K}_{\tilde{u}}(0) \times\{\tilde{u}\}, \quad \mathcal{A}=\bigcup_{\tilde{u} \in H(\tilde{u} \#)} \mathcal{K}_{\tilde{u}}(0) .
$$

Remark 4.3. Note that $\mathcal{A}$ is bounded in $H^{2}(\Omega) \times H_{0}^{1}(\Omega)$, thanks to Lemma 4.1 ,

REMARK 4.4. The translation compactness assumption on $\tilde{u}^{\#}$ can be weakened according to 34.

5. Convergence to equilibria. In this section we prove that any solution to problem (2.2) converges to a single equilibrium as time goes to infinity, provided that the boundary datum $\tilde{u}$ suitably converges to some time-independent function $\tilde{u}^{*}$.

In order to demonstrate our result we need the following assumptions:

(U1) $\tilde{u} \in C\left([0, \infty), H^{\frac{3}{2}}(\Gamma)\right), \tilde{u}_{t} \in L^{1}\left(0, \infty, H^{\frac{3}{2}}(\Gamma)\right)$;

(U2) there are constants $\gamma>0$ and $C>0$ such that

$$
\left\|\tilde{u}_{t}(t)\right\|_{H^{-\frac{1}{2}}(\Gamma)} \leq C t^{-2-\gamma}, \quad \forall t \geq 1 .
$$

Note that the straightforward consequences of them are (cf. (2.1))

$$
\hat{u} \in C\left([0, \infty), H^{2}(\Omega)\right)
$$

and

$$
\left\|\hat{u}_{t}(t)\right\| \leq C t^{-2-\gamma}, \quad \forall t \geq 1 .
$$

In addition, assumption (U1) implies that there exists $\tilde{u}^{*} \in H^{\frac{3}{2}}(\Gamma)$ such that $\tilde{u}(t)$ strongly converges to $\tilde{u}^{*}$ in $H^{\frac{3}{2}}(\Gamma)$ as $t$ goes to $\infty$. Consequently, $\hat{u}(t)$ converges to a function $\hat{u}^{*}$ in $H^{2}(\Omega)$. 
REMARK 5.1. Observe that (5.1) implies

$$
\int_{t}^{\infty}\left\|\tilde{u}_{t}(\tau)\right\|_{H^{-\frac{1}{2}}(\Gamma)} d \tau \leq C t^{-1-\gamma}, \quad \forall t \geq 1,
$$

and, accordingly,

$$
\int_{t}^{\infty}\left\|\hat{u}_{t}(\tau)\right\| d \tau \leq C t^{-1-\gamma}, \quad \forall t \geq 1 .
$$

Apparently, (5.1) is stronger than (5.4). Actually, we only need (5.4) to get the convergence rate in lower order norm (see, for instance, (5.75)-(5.78) below). When we want to establish the convergence rate in a higher-order norm (see (5.27)), then it is natural to require more on the Dirichlet datum $\tilde{u}$.

Let us begin with this preliminary lemma on some properties of the global solution to (2.2). From now on we take $\tau=0$.

Lemma 5.1. Under the assumptions (U1), (U2), for any $\left(f_{0}, u_{0}\right) \in \mathcal{H}$, problem (2.2) admits a unique global solution $(f, u)$ such that

$$
f \in C\left([0, \infty), H^{1}(\Omega)\right), \quad f_{t} \in L^{2}\left([0, \infty), L^{2}(\Omega)\right)
$$

and

$$
u \in C\left([0, \infty), L^{2}(\Omega)\right) \cap L^{2}\left([0, \infty), H_{0}^{1}(\Omega)\right) .
$$

Moreover, there exist $f_{\infty} \in H^{1}(\Omega)$ and $u_{\infty} \in L^{2}(\Omega)$ such that

$$
\lim _{t \rightarrow \infty} E(f(t), u(t), \hat{u}(t))=E\left(f_{\infty}, u_{\infty}, \hat{u}^{*}\right),
$$

where $E(f, u, \hat{u})$ is defined by the following:

$$
E(f, u, \hat{u}):=\frac{1}{2 k^{2}} \int_{\Omega}|\nabla f|^{2} d x+\int_{\Omega}\left(\frac{1}{4} f^{4}+\frac{\hat{u}-1}{2} f^{2}\right) d x+\frac{1}{2} \int_{\Omega} u^{2} d x .
$$

Proof. It follows from Lemma 4.1 that, for all $t \geq 1$, we have

$$
\|f(t)\|_{H^{2}} \leq C, \quad\|u(t)\|_{H_{0}^{1}} \leq C .
$$

Thus, there is $f_{\infty} \in H^{2}(\Omega)$ and $u_{\infty} \in H^{1}(\Omega)$, and an unbounded increasing sequence $\left\{t_{n}\right\}_{n=1}^{\infty}$ such that

$$
\lim _{t_{n} \rightarrow \infty}\left\|f\left(t_{n}\right)-f_{\infty}\right\|_{H^{1}}=0, \quad \lim _{t_{n} \rightarrow \infty}\left\|u\left(t_{n}\right)-u_{\infty}\right\|=0
$$

i.e., the $\omega$-limit set of $\left(f_{0}, u_{0}\right)$ is nonempty. Furthermore, we deduce from (3.6) with $\varepsilon=0$ the following identity:

$$
\begin{aligned}
\frac{d}{d t} E(f, u, \hat{u})+\left\|f_{t}\right\|^{2}+\eta\|\nabla u\|^{2} & =\frac{1}{2} \int_{\Omega} f^{2} \hat{u}_{t} d x-\int_{\Omega} \hat{u}_{t} u d x \\
& \leq \frac{1}{2}\|f\|_{L^{4}}^{2}\left\|\hat{u}_{t}\right\|+\|u\|\left\|\hat{u}_{t}\right\| \\
& \leq C\left\|\hat{u}_{t}\right\|,
\end{aligned}
$$

where $C$ is a constant depending only on $\left\|f_{0}\right\|_{H^{1}},\left\|u_{0}\right\|$, and $\|\tilde{u}\|_{L^{\infty}\left(0, \infty ; H^{-\frac{1}{2}}(\Gamma)\right)}$. On the other hand, by the Young inequality, we infer from (5.9) that

$$
E(f, u, \hat{u}) \geq \int_{\Omega}\left(\frac{1}{2 k^{2}}|\nabla f|^{2}+\frac{1}{8} f^{4}+\frac{1}{2} u^{2}\right) d x-C\left(1+\|\hat{u}\|^{2}\right) ;
$$


that is, $E$ is bounded from below. Thus, (5.6) and (5.7) follow by integrating (5.12) with respect to $t$ from 0 to $\infty$. As a further consequence of (5.12), we deduce, for $0 \leq t_{2} \leq t_{1}$, that

$$
\begin{gathered}
\left|E\left(f\left(t_{1}\right), u\left(t_{1}\right), \hat{u}\left(t_{1}\right)\right)-E\left(f\left(t_{2}\right), u\left(t_{2}\right), \hat{u}\left(t_{2}\right)\right)\right| \\
\leq \int_{t_{2}}^{t_{1}}\left\|f_{t}\right\|^{2}+\eta\|\nabla u\|^{2} d t+C \int_{t_{2}}^{t_{1}}\left\|\hat{u}_{t}(t)\right\| d t .
\end{gathered}
$$

The conclusion then follows from (5.4), (5.11) and (5.14).

Let us now introduce the functional

$$
\Upsilon(f):=\frac{1}{2 k^{2}} \int_{\Omega}|\nabla f|^{2} d x+\int_{\Omega}\left(\frac{1}{4} f^{4}+\frac{\hat{u}^{*}-1}{2} f^{2}\right) d x
$$

and the set

$$
\mathcal{S}:=\left\{v \in H^{2}(\Omega)\left|\frac{1}{k^{2}} \Delta v-\left(v^{2}-1+\hat{u}^{*}\right) v=0, \quad \partial_{\nu} v\right|_{\Gamma}=0\right\} .
$$

Arguing as, for instance, in [39, Section 3], we deduce that

Lemma 5.2. $\mathcal{S}$ is nonempty. If $v \in \mathcal{S}$, then $v$ is a critical point of the functional $\Upsilon(f)$ over $H^{1}(\Omega)$. Conversely, if $v \in H^{1}(\Omega)$ is a critical point of $\Upsilon(f)$ on $H^{1}(\Omega)$, then $v \in \mathcal{S}$.

We can now show that the $\omega$-limit set of $\left(f_{0}, u_{0}\right)$ is a subset of $\mathcal{S} \times\{0\}$. Indeed we have

Lemma 5.3. Let $\left(f_{\infty}, u_{\infty}\right)$ be a cluster point of the global solution $(f(t), u(t))$ to (2.2) as $t$ goes to $\infty$. Then $f_{\infty} \in \mathcal{S}$ and $u_{\infty}=0$.

Proof. Observe first that, for $t \geq 1$,

$$
\begin{aligned}
\frac{\eta}{2} \frac{d}{d t}\|\nabla u\|^{2}+\left\|u_{t}\right\|^{2} & =\int_{\Omega} f f_{t} u_{t} d x-\int_{\Omega} \hat{u}_{t} u_{t} d x \\
& \leq \frac{1}{2}\left\|f f_{t}\right\|^{2}+\left\|\hat{u}_{t}\right\|^{2}+\frac{1}{2}\left\|u_{t}\right\|^{2} \\
& \leq C\left\|f_{t}\right\|^{2}+\left\|\hat{u}_{t}\right\|^{2}+\frac{1}{2}\left\|u_{t}\right\|^{2},
\end{aligned}
$$

where in the last step we have used Lemma 4.1 and the Sobolev embedding $H^{2}(\Omega) \hookrightarrow$ $C^{0}(\bar{\Omega})(n \leq 3)$. On the other hand, we know that (cf. (5.6))

$$
\int_{0}^{\infty}\left\|f_{t}(t)\right\|^{2} d t<\infty
$$

Then, by (5.4) and [41, Lemma 6.2.1], it follows from (5.17) that

$$
\lim _{t \rightarrow \infty}\|\nabla u(t)\|=0,
$$

which entails, owing to the Dirichlet boundary condition and the Poincaré inequality,

$$
\lim _{t \rightarrow \infty}\|u(t)\|_{H^{1}(\Omega)}=0,
$$

so that $u_{\infty}=0$ (see (5.11) $)$. 
To show that $f_{\infty} \in \mathcal{S}$, we apply the argument devised in [28. Up to a subsequence, we can assume $t_{n+1} \geq t_{n}+1, n \in \mathbb{N}$, without loss of generality. Integrating (5.12) on the time interval $\left[t_{n}, t_{n+1}\right]$ we obtain

$$
\begin{aligned}
& E\left(f\left(t_{n+1}\right), u\left(t_{n+1}\right), \hat{u}\left(t_{n+1}\right)\right)-E\left(f\left(t_{n}\right), u\left(t_{n}\right), \hat{u}\left(t_{n}\right)\right) \\
+ & \int_{t_{n}}^{t_{n+1}}\left(\left\|f_{t}(\tau)\right\|^{2}+\eta\|\nabla u(\tau)\|^{2}\right) d \tau \\
\leq & C \int_{t_{n}}^{t_{n+1}}\left\|\hat{u}_{t}(\tau)\right\| d \tau \leq C \int_{t_{n}}^{\infty}\left\|\hat{u}_{t}(\tau)\right\| d \tau .
\end{aligned}
$$

It follows from (5.4) that

$$
\begin{aligned}
& \int_{0}^{1}\left(\left\|f_{t}\left(t_{n}+t\right)\right\|^{2}+\eta\left\|\nabla u\left(t_{n}+t\right)\right\|^{2}\right) d t \\
\leq & \int_{0}^{t_{n+1}-t_{n}}\left(\left\|f_{t}\left(t_{n}+t\right)\right\|^{2}+\eta\left\|\nabla u\left(t_{n}+t\right)\right\|^{2}\right) d t \rightarrow 0,
\end{aligned}
$$

as $n \rightarrow \infty$. Thus we have

$$
\left\|f\left(t_{n}+t\right)-f\left(t_{n}+t^{\prime}\right)\right\| \rightarrow 0, \quad \text { uniformly for } t, t^{\prime} \in[0,1] .
$$

From (5.10), which implies the precompactness of $f(t)$ in $H^{1}(\Omega)$, it follows that

$$
\lim _{n \rightarrow \infty}\left\|f\left(t_{n}+t\right)-f_{\infty}\right\|_{H^{1}}=0, \quad \forall t \in[0,1],
$$

and by the mean value theorem, it follows from (5.22) that there are $t_{n}^{*} \in[0,1]$ such that

$$
\left\|f_{t}\left(t_{n}^{\prime}\right)\right\| \rightarrow 0
$$

where $t_{n}^{\prime}=t_{n}+t_{n}^{*}$.

Hence, recalling (U1), (5.20), (5.22), and (5.24), we can compute the first equation of (2.2) at $t=t_{n}^{\prime}$ and pass to the limit as $n$ goes to $\infty$. Thus we get

$$
\left\|\frac{1}{k^{2}} \Delta f_{\infty}-\left(f_{\infty}^{2}-1+\hat{u}^{*}\right) f_{\infty}\right\|_{\left(H^{1}\right)^{\prime}}=0
$$

which implies $f_{\infty} \in \mathcal{S}$, because of Lemma 5.2

After these preliminary considerations, we can state the main result of the present section. That is,

Theorem 5.1. Assume that (U1) and (U2) are satisfied. If $\left(f_{0}, u_{0}\right) \in \mathcal{H}$, then the corresponding global solution $(f(t), u(t))$ given by Theorem 2.1 has a unique cluster point $\left(f_{\infty}, 0\right)$. Moreover, there is $\theta \in\left(0, \frac{\gamma}{2(1+\gamma)}\right)$ such that the following convergence estimate holds:

$$
\left\|f(t)-f_{\infty}\right\|_{H^{1}}+\|u(t)\|_{H^{1}} \leq C t^{-\sigma}, \quad t \geq t_{0}^{*},
$$

for some $t_{0}^{*} \geq 1$, where

$$
\sigma:=\frac{\theta}{1-2 \theta} .
$$

REMARK 5.2. Since $u_{\infty}=0$, we can easily see that

$$
E\left(f_{\infty}, u_{\infty}, \hat{u}^{*}\right)=\Upsilon\left(f_{\infty}\right) .
$$


The main tool to demonstrate Theorem 5.1 is a suitable version of the LojasiewiczSimon inequality as follows.

Lemma 5.4. Let $f_{\infty}$ be a critical point of $\Upsilon(f)$. Then there exist constants $\theta \in\left(0, \frac{1}{2}\right)$ and $\beta>0$ depending on $f_{\infty}$ such that, for any $f \in H^{2}(\Omega)$ satisfying $\left\|f-f_{\infty}\right\|_{H^{1}}<\beta$, we have

$$
\left\|-\frac{1}{k^{2}} \Delta f+f^{3}+\left(\hat{u}^{*}-1\right) f\right\|_{\left(H^{1}\right)^{\prime}} \geq\left|\Upsilon(f)-\Upsilon\left(f_{\infty}\right)\right|^{1-\theta} .
$$

Since the proof of this lemma is analogous to the arguments developed, for instance, in 27, 29] with minor modifications, we omit the details. Moreover, we observe that, via the Poincare inequality, it is easy to draw the following conclusion.

Lemma 5.5. Let $f_{\infty}$ be a critical point of $\Upsilon(f)$. Then there exist constants $\theta \in\left(0, \frac{1}{2}\right)$ and $\beta>0$ depending on $f_{\infty}$ such that, for any $f \in H^{2}(\Omega)$ satisfying $\left\|f-f_{\infty}\right\|_{H^{1}}<\beta$ and $u \in H_{0}^{1}(\Omega)$, we have

$$
\left\|-\frac{1}{k^{2}} \Delta f+f^{3}+\left(\hat{u}^{*}-1\right) f\right\|_{\left(H^{1}\right)^{\prime}}+C\|\nabla u\| \geq\left|E\left(f, u, \hat{u}^{*}\right)-\Upsilon\left(f_{\infty}\right)\right|^{1-\theta} .
$$

We are now in a position to prove Theorem 5.1.

Proof. Let us prove the convergence result first. Recalling Lemma 5.3, let $\left(f_{\infty}, 0\right)$ be a cluster point of the global solution $(f, u)$. Then $f_{\infty} \in \mathcal{S}$, and there exists an unbounded increasing sequence $\left\{t_{n}\right\}_{n \in \mathbb{N}}$ such that

$$
\lim _{t_{n} \rightarrow \infty}\left\|f\left(t_{n}\right)-f_{\infty}\right\|_{H^{1}}=0 .
$$

Integrating (5.12) with respect to time over $[t, \infty)$, with $t \geq 1$, from Lemma 5.1 and (5.29) we infer that

$$
\begin{aligned}
& \left|E(f(t), u(t), \hat{u}(t))-\Upsilon\left(f_{\infty}\right)\right| \\
\geq & \int_{t}^{\infty}\left(\left\|f_{t}(\tau)\right\|^{2}+\eta\|\nabla u(\tau)\|^{2}\right) d \tau-C \int_{t}^{\infty}\left\|\hat{u}_{t}(\tau)\right\| d \tau .
\end{aligned}
$$

Suppose now that there is a $t_{0} \geq 1$ such that, for all $t \geq t_{0}, f$ satisfies the condition of Lemma [5.5] i.e., $\left\|f(t)-f_{\infty}\right\|_{H^{1}}<\beta$. We recall that the existence of such a $t_{0}$ can be obtained by a contradiction argument (see, for instance, 24]; cf. also 28]).

Moreover, because of (5.8) and (5.29), we can take $t_{0}$ large enough such that

$$
\left|E(f(t), u(t), \hat{u}(t))-\Upsilon\left(f_{\infty}\right)\right| \leq 1, \quad \forall t \geq t_{0} .
$$

Now following the argument devised in [28, we introduce the following functions:

$$
Z(t)^{2}:=\left\|f_{t}(t)\right\|^{2}+\eta\|\nabla u(t)\|^{2}, \quad \zeta(t):=\int_{t}^{\infty}\left\|\hat{u}_{t}(\tau)\right\| d \tau, \quad \forall t \geq t_{0},
$$

and we observe that (U2) entails

$$
0 \leq \zeta(t) \leq z(t):=C t^{-1-\gamma}, \quad \forall t \geq t_{0} .
$$


We recall that, owing to Lemma 5.5. we have, for all $t \geq t_{0}$,

$$
\left\|\left(-\frac{1}{k^{2}} \Delta f+f^{3}+\left(\hat{u}^{*}-1\right) f\right)(t)\right\|_{\left(H^{1}\right)^{\prime}}+C\|\nabla u(t)\| \geq\left|E\left(f(t), u(t), \hat{u}^{*}\right)-\Upsilon\left(f_{\infty}\right)\right|^{1-\theta} .
$$

On the other hand, there holds

$$
\begin{aligned}
& \left|E(f(t), u(t), \hat{u}(t))-\Upsilon\left(f_{\infty}\right)\right|^{1-\theta} \\
\leq & \left|E\left(f(t), u(t), \hat{u}^{*}\right)-\Upsilon\left(f_{\infty}\right)\right|^{1-\theta}+\left|E(f(t), u(t), \hat{u}(t))-E\left(f(t), u(t), \hat{u}^{*}\right)\right|^{1-\theta} \\
\leq & \left|E\left(f(t), u(t), \hat{u}^{*}\right)-\Upsilon\left(f_{\infty}\right)\right|^{1-\theta}+C\left|\int_{\Omega}\left(\hat{u}(t)-\hat{u}^{*}\right) f^{2}(t) d x\right|^{1-\theta}
\end{aligned}
$$

and

$$
\left|\int_{\Omega}\left(\hat{u}(t)-\hat{u}^{*}\right) f^{2}(t) d x\right|^{1-\theta} \leq\|f(t)\|_{L^{4}(\Omega)}^{2(1-\theta)}\left\|\hat{u}(t)-\hat{u}^{*}\right\|^{1-\theta} \leq C \zeta(t)^{1-\theta} .
$$

Moreover, we have

$$
\begin{aligned}
& \left\|-\frac{1}{k^{2}} \Delta f+f^{3}+\left(\hat{u}^{*}(x)-1\right) f\right\|_{\left(H^{1}\right)^{\prime}}+C\|\nabla u\| \\
= & \left\|-\frac{1}{k^{2}} \Delta f+f^{3}+(u+\hat{u}-1) f-u f+\left(\hat{u}^{*}-\hat{u}\right) f\right\|_{\left(H^{1}\right)^{\prime}}+C\|\nabla u\| \\
\leq & \left\|f_{t}\right\|_{\left(H^{1}\right)^{\prime}}+\|u f\|_{\left(H^{1}\right)^{\prime}}+\left\|\left(\hat{u}^{*}-\hat{u}\right) f\right\|_{\left(H^{1}\right)^{\prime}}+C\|\nabla u\| \\
\leq & \left\|f_{t}\right\|_{\left(H^{1}\right)^{\prime}}+C\|\nabla u\|+C \zeta .
\end{aligned}
$$

Hence, inequalities (5.33)-(5.40) imply that

$$
\begin{aligned}
\left(\int_{t}^{\infty} Z(\tau)^{2} d \tau-z(t)\right)^{1-\theta} & \leq\left|E(f(t), u(t), \hat{u}(t))-\Upsilon\left(f_{\infty}\right)\right|^{1-\theta} \\
& \leq\left|E\left(f(t), u(t), \hat{u}^{*}\right)-\Upsilon\left(f_{\infty}\right)\right|^{1-\theta}+C z(t)^{1-\theta} \\
& \leq C\left(Z(t)+z(t)+z(t)^{1-\theta}\right) \\
& \leq C\left(Z(t)+z(t)^{1-\theta}\right) .
\end{aligned}
$$

Thus, for all $t \geq t_{0}$, we obtain

$$
\int_{t}^{\infty} Z(\tau)^{2} d \tau-z(t) \leq C\left(Z(t)+z(t)^{1-\theta}\right)^{\frac{1}{1-\theta}} .
$$

Taking (5.36) into account, we can choose $\theta$ such that

$$
0<2 \theta<1-\frac{1}{1+\gamma}<1
$$

This choice yields

$$
\int_{t}^{\infty} z(\tau)^{2(1-\theta)} d \tau \leq C z(t), \quad \forall t \geq t_{0} .
$$

Therefore we can deduce from (5.42) and (5.44) that

$$
\int_{t}^{\infty}\left(Z(\tau)+z(\tau)^{1-\theta}\right)^{2} d \tau \leq C\left(Z(t)+z(t)^{1-\theta}\right)^{\frac{1}{1-\theta}},
$$


and from [28, Lemma 4.1] we infer

$$
\int_{0}^{\infty} Z(\tau) d \tau<\infty
$$

which gives

$$
\lim _{t \rightarrow \infty}\left\|f(t)-f_{\infty}\right\|=0
$$

Hence, thanks to (5.32), we get

$$
\lim _{t \rightarrow \infty}\left\|f(t)-f_{\infty}\right\|_{H^{1}}=0
$$

We now know that the global solution $(f(t), u(t))$ converges to $\left(f_{\infty}, 0\right)$ in $\left(H^{1}(\Omega)\right)^{2}$ as $t$ goes to $\infty$. It remains to prove (5.27). We first prove the convergence rate estimate for the $L^{2}$-norm of $f(t)-f_{\infty}$ and the $H^{-1}$-norm of $u(t)$.

Let $t_{0} \geq 1$ such that for all $t \geq t_{0}, f$ satisfies the condition of Lemma 5.5 i.e., $\left\|f-f_{\infty}\right\|_{H^{1}(\Omega)}<\beta$. It follows from (5.12) that

$$
\frac{d}{d t} E(f, u, \hat{u}) \leq-\left\|f_{t}\right\|^{2}-\eta\|\nabla u\|^{2}+C_{8}\left\|\hat{u}_{t}\right\| .
$$

We take advantage of the technique employed in [11, 24], and we divide the time interval $J:=\left[t_{0}, \infty\right)$ into two parts,

$$
\begin{gathered}
J_{1}=\left\{t \in J:\left\|f_{t}(t)\right\|+\|\nabla u(t)\|>\left(\int_{t}^{\infty}\left\|\hat{u}_{t}(\tau)\right\| d \tau\right)^{1-\theta}\right\}, \\
J_{2}=J \backslash J_{1} .
\end{gathered}
$$

Then we define

$$
\Phi(t):=E(f(t), u(t), \hat{u}(t))-\Upsilon\left(f_{\infty}\right)+\left(C_{8}+1\right) \int_{t}^{\infty}\left\|\hat{u}_{t}(\tau)\right\| d \tau
$$

and observe that

$$
\frac{d}{d t} \Phi(t) \leq-\left\|f_{t}(t)\right\|^{2}-\eta\|\nabla u(t)\|^{2}-\left\|\hat{u}_{t}(t)\right\| \leq 0 .
$$

Besides, there holds

$$
\lim _{t \rightarrow \infty} \Phi(t)=0 .
$$

If there exists $T_{1} \geq t_{0}$, such that $\Phi\left(T_{1}\right)=0$, a time integration of (5.53) on $\left(T_{1}, \infty\right)$ yields

$$
\left\|f_{t}(t)\right\|=\|\nabla u(t)\|=\left\|\hat{u}_{t}(t)\right\| \equiv 0, \quad t \geq T_{1},
$$

which implies

$$
f(t)=f_{\infty}, \quad u(t)=0, \quad \hat{u}(t)=\hat{u}^{*}, \quad t \geq T_{1},
$$

and we are done. Thus, in what follows we can assume

$$
\Phi(t)>0, \quad \forall t \geq 1 .
$$


From Lemma 5.5, (5.38), and (5.39) we deduce that

$$
\begin{aligned}
& \left|E(f(t), u(t), \hat{u}(t))-\Upsilon\left(f_{\infty}\right)\right|^{1-\theta} \\
\leq & \left|E\left(f(t), u(t), \hat{u}^{*}\right)-\Upsilon\left(f_{\infty}\right)\right|^{1-\theta}+C\left|\int_{\Omega}\left(\hat{u}(t)-\hat{u}^{*}\right) f^{2}(t) d x\right|^{1-\theta} \\
\leq & \left\|f_{t}(t)\right\|_{\left(H^{1}(\Omega)\right)^{\prime}}+C\|\nabla u(t)\|+C\left(\int_{t}^{\infty}\left\|\hat{u}_{t}(\tau)\right\| d \tau\right)^{1-\theta} .
\end{aligned}
$$

Since $J_{1}$ is an open set, there exists a countable family of disjoint open sets $\left(a_{n}, b_{n}\right)$ such that $J_{1}=\bigcup_{n=1}^{\infty}\left(a_{n}, b_{n}\right)$. If $J_{1}$ is a bounded set, then there is $t^{*}>0$ such that $J_{2} \subset\left(t^{*},+\infty\right)$. It follows from the definition of $J_{2}$ and assumption (U2) that, for $t \geq t^{*}$,

$$
\left\|f_{t}(t)\right\|+\|\nabla u(t)\| \leq\left(\int_{t}^{\infty}\left\|\hat{u}_{t}(\tau)\right\| d \tau\right)^{1-\theta} \leq C t^{-(1+\gamma)(1-\theta)}
$$

Thus, for $t \geq t^{*}$,

$$
\left\|f(t)-f_{\infty}\right\| \leq \int_{t}^{\infty}\left\|f_{t}(\tau)\right\| d \tau \leq C t^{1-(1+\gamma)(1-\theta)}, \quad\|u(t)\|_{H^{1}} \leq C t^{-(1+\gamma)(1-\theta)} .
$$

From (5.43) we infer that

$$
(1+\gamma)(1-\theta)-1>\frac{\gamma}{2}>\frac{\theta}{1-2 \theta},
$$

and the proof is done. Thus in what follows we only consider the case that $J_{1}$ is unbounded. On $J_{1}$ we have

$$
\begin{aligned}
|\Phi(t)|^{1-\theta} & \leq\left|E(f(t), u(t), \hat{u}(t))-\Upsilon\left(f_{\infty}\right)\right|^{1-\theta}+\left(\int_{t}^{\infty}\left(C_{8}+1\right)\left\|\hat{u}_{t}(\tau)\right\| d \tau\right)^{1-\theta} \\
& \leq C\left(\left\|f_{t}(t)\right\|+\|\nabla u(t)\|\right) .
\end{aligned}
$$

Hence, for every $t \in J_{1}$, it follows from (5.53) that

$$
\frac{d}{d t} \Phi(t)+C \Phi(t)^{2(1-\theta)} \leq 0 .
$$

This yields that, for any $t \in\left(a_{n}, b_{n}\right) \subset J_{1}$,

$$
\Phi(t) \leq\left(\Phi\left(a_{n}\right)^{2 \theta-1}+C(1-2 \theta)\left(t-a_{n}\right)\right)^{-\frac{1}{1-2 \theta}} .
$$

From the definition of $J_{1}$, (5.4), and (5.61), we infer

$$
\left|\Phi\left(a_{n}\right)\right| \leq C\left(\left\|f_{t}\left(a_{n}\right)\right\|+\left\|\nabla u\left(a_{n}\right)\right\|\right)^{\frac{1}{1-\theta}}=C \int_{a_{n}}^{\infty}\left\|\hat{u}_{t}(\tau)\right\| d \tau \leq C a_{n}^{-1-\gamma},
$$

and (5.43) implies

$$
(1+\gamma)(1-2 \theta)>1 .
$$

As a result, we can choose $n^{*} \in \mathbb{N}$ large enough such that

$$
\Phi\left(a_{n^{*}}\right)^{2 \theta-1}-C(1-2 \theta) a_{n^{*}} \geq a_{n^{*}}^{(1+\gamma)(1-2 \theta)}-C(1-2 \theta) a_{n^{*}} \geq 0 .
$$

Therefore, (5.63) and (5.66) entail

$$
\Phi(t) \leq C t^{-\frac{1}{1-2 \theta}}, \quad \forall t \in\left(a_{n^{*}}, b_{n^{*}}\right) .
$$


Thus, we have

$$
\Phi(t) \leq C t^{-\frac{1}{1-2 \theta}}, \quad \forall t \in\left(a_{n^{*}}, \infty\right) \cap J_{1} .
$$

On the other hand, from $J_{1}$ we deduce by (5.53) and (5.61) that

$$
-\frac{d}{d t} \Phi(t)^{\theta}=-\theta|\Phi(t)|^{-1+\theta} \frac{d}{d t} \Phi(t) \geq C\left(\left\|f_{t}(t)\right\|+\|\nabla u(t)\|\right) .
$$

Consequently, for any $t \in\left(a_{n^{*}}, \infty\right) \cap J_{1}$, we find

$$
\int_{(t, \infty) \cap J_{1}}\left(\left\|f_{t}(\tau)\right\|+\|\nabla u(\tau)\|\right) d \tau \leq C \Phi(t)^{\theta} \leq C t^{-\frac{\theta}{1-2 \theta}}
$$

while on the measurable set $J_{2}$ we have

$$
\int_{(t, \infty) \cap J_{2}}\left(\left\|f_{t}(\tau)\right\|+\|\nabla u(\tau)\|\right) d \tau \leq \int_{t}^{\infty} z(\tau)^{1-\theta} d \tau \leq C t^{1-(1+\gamma)(1-\theta)} .
$$

Note that, recalling (5.43), there holds

$$
1-(1+\gamma)(1-\theta) \leq 1-\frac{1-\theta}{1-2 \theta}=-\frac{\theta}{1-2 \theta} .
$$

Thus, we obtain

$$
\int_{t}^{\infty}\left(\left\|f_{t}(\tau)\right\|+\|\nabla u(\tau)\|\right) d \tau \leq C t^{-\sigma}, \quad t \geq t_{0}^{*}
$$

with

$$
\sigma=\frac{\theta}{1-2 \theta}, \quad t_{0}^{*}=\max \left\{t_{0}, a_{n^{*}}\right\} .
$$

It follows from (5.73) that

$$
\left\|f(t)-f_{\infty}\right\| \leq C t^{-\sigma}, \quad t \geq t_{0}^{*} \geq 1 .
$$

From the equation for $u$ we deduce

$$
\|\nabla u\|=\left\|u_{t}-f f_{t}-\hat{u}_{t}\right\|_{H^{-1}} \geq\left\|u_{t}\right\|_{H^{-1}}-\left\|f f_{t}\right\|_{H^{-1}}-\left\|\hat{u}_{t}\right\|_{H^{-1}} .
$$

Hence, we get, for all $t \geq t_{0}^{*}$,

$$
\begin{aligned}
& \int_{t}^{\infty}\left\|u_{t}(\tau)\right\|_{H^{-1}} d \tau \\
\leq & \int_{t}^{\infty}\left(\|f(\tau)\|_{L^{\infty}}\left\|f_{t}(\tau)\right\|+\|\nabla u(\tau)\|+\left\|\hat{u}_{t}(\tau)\right\|\right) d \tau \leq C t^{-\sigma},
\end{aligned}
$$

which implies

$$
\|u(t)\|_{H^{-1}} \leq C t^{-\sigma}, \quad t \geq t_{0}^{*} \geq 1 .
$$

We have thus proven the estimate

$$
\left\|f(t)-f_{\infty}\right\|+\|u\|_{H^{-1}} \leq C t^{-\sigma}, \quad t \geq t_{0}^{*} \geq 1 .
$$

We now proceed to demonstrate estimate (5.27) (see, e.g., [39] for a similar argument). Setting $\bar{f}:=f-f_{\infty}$ and $\bar{u}:=u$, we have that $\bar{f}_{t}=f_{t}$ and $(\bar{f}, \bar{u})$ solves the system

$$
\begin{gathered}
\bar{f}_{t}=\frac{1}{k^{2}} \Delta \bar{f}-u f-\left(\hat{u} f-\hat{u}^{*} f_{\infty}\right)+\bar{f}-f^{3}+f_{\infty}^{3}, \\
\bar{u}_{t}-f f_{t}=\eta \Delta \bar{u}-\hat{u}_{t} .
\end{gathered}
$$


We multiply the first equation by $\bar{f}_{t}+\varepsilon \bar{f}$ and the second equation by $\bar{u}$, integrate on $\Omega$, and then add the results together to obtain

$$
\begin{aligned}
& \frac{d}{d t}\left[\frac{1}{2}\|\bar{u}\|^{2}+\frac{1}{2 k^{2}}\|\nabla \bar{f}\|^{2}+\frac{\varepsilon}{2}\|\bar{f}\|^{2}\right]+\eta\|\nabla \bar{u}\|^{2}+\left\|\bar{f}_{t}\right\|^{2}+\frac{\varepsilon}{k^{2}}\|\nabla \bar{f}\|^{2} \\
= & \varepsilon \int_{\Omega} \bar{f}^{2} d x-\varepsilon \int_{\Omega} \bar{u} f \bar{f} d x-\varepsilon \int_{\Omega}\left(\hat{u} f-\hat{u}^{*} f_{\infty}\right) \bar{f} d x \\
& -\varepsilon \int_{\Omega} \bar{f}^{2}\left(f^{2}+f f_{\infty}+f_{\infty}^{2}\right) d x-\int_{\Omega}\left(\hat{u} f-\hat{u}^{*} f_{\infty}\right) \bar{f}_{t} d x \\
& +\int_{\Omega} \bar{f} \bar{f}_{t} d x-\int_{\Omega} \bar{f} \bar{f}_{t}\left(f^{2}+f f_{\infty}+f_{\infty}^{2}\right) d x-\int_{\Omega} \hat{u}_{t} \bar{u} d x .
\end{aligned}
$$

Let us estimate the terms on the right hand side. We have

$$
\begin{gathered}
\varepsilon\left|\int_{\Omega} \bar{u} f \bar{f} d x\right| \leq \varepsilon\|\bar{u}\|\|f\|_{L^{\infty}}\|\bar{f}\| \leq \varepsilon\|\bar{u}\|^{2}+C \varepsilon\|\bar{f}\|^{2}, \\
\varepsilon\left|\int_{\Omega}\left(\hat{u} f-\hat{u}^{*} f_{\infty}\right) \bar{f} d x\right| \leq \varepsilon\left(\left\|\hat{u}-\hat{u}^{*}\right\|\|\bar{f}\|\|f\|_{L^{\infty}}+\left\|\hat{u}^{*}\right\|_{L^{\infty}}\|\bar{f}\|^{2}\right) \\
\leq C \varepsilon\left(\left\|\hat{u}-\hat{u}^{*}\right\|^{2}+\|\bar{f}\|^{2}\right), \\
\varepsilon\left|\int_{\Omega} \bar{f}^{2}\left(f^{2}+f f_{\infty}+f_{\infty}^{2}\right) d x\right| \leq \varepsilon\|\bar{f}\|^{2}\left\|f^{2}+f f_{\infty}+f_{\infty}^{2}\right\|_{L^{\infty}} \leq C \varepsilon\|\bar{f}\|^{2}, \\
\left|\int_{\Omega}\left(\hat{u} f-\hat{u}^{*} f_{\infty}\right) \bar{f}_{t} d x\right| \\
\leq\|\hat{u}\|_{L^{\infty}}\|\bar{f}\|\left\|\bar{f}_{t}\right\|+\left\|f_{\infty}\right\|_{L^{\infty}}\left\|\hat{u}-\hat{u}^{*}\right\|\left\|\bar{f}_{t}\right\| \\
\leq \frac{1}{4}\left\|\bar{f}_{t}\right\|^{2}+C\|\bar{f}\|^{2}+C\left\|\hat{u}-\hat{u}^{*}\right\|^{2}, \\
\left|\int_{\Omega} \bar{f} \bar{f}_{t}\left(f^{2}+f f_{\infty}+f_{\infty}^{2}\right) d x\right| \leq\left\|\bar{f}_{t}\right\|\|\bar{f}\|\left\|f^{2}+f f_{\infty}+f_{\infty}^{2}\right\|_{L^{\infty}} \\
\leq \frac{1}{4}\left\|\bar{f}_{t}\right\|^{2}+C\|\bar{f}\|^{2} .
\end{gathered}
$$

Combining the above estimates together, we deduce

$$
\begin{aligned}
& \frac{d}{d t}\left[\frac{1}{2}\|\bar{u}\|^{2}+\frac{1}{2 k^{2}}\|\nabla \bar{f}\|^{2}+\frac{\varepsilon}{2}\|\bar{f}\|^{2}\right]+\frac{\eta}{2}\|\nabla \bar{u}\|^{2}+\frac{1}{8}\left\|\bar{f}_{t}\right\|^{2}+\frac{\varepsilon}{k^{2}}\|\nabla \bar{f}\|^{2} \\
\leq & C \varepsilon\|\bar{u}\|^{2}+C\left(\|\bar{f}\|^{2}+\left\|\hat{u}-\hat{u}^{*}\right\|^{2}\right) .
\end{aligned}
$$

Since $u=\bar{u}$ satisfies the homogeneous Dirichlet boundary condition, by the Poincaré inequality we have

$$
\|\bar{u}\| \leq C\|\nabla \bar{u}\|^{2}
$$

Thus, by taking $\varepsilon$ small enough, we deduce from (5.89) that

$$
\begin{aligned}
& \frac{d}{d t}\left[\frac{1}{2}\|\bar{u}\|^{2}+\frac{1}{2 k^{2}}\|\nabla \bar{f}\|^{2}+\frac{\varepsilon}{2}\|\bar{f}\|^{2}\right]+\frac{\eta}{4}\|\nabla \bar{u}\|^{2}+\frac{1}{8}\left\|\bar{f}_{t}\right\|^{2}+\frac{\varepsilon}{k^{2}}\|\nabla \bar{f}\|^{2} \\
& \leq C\left(\|\bar{f}\|^{2}+\left\|\hat{u}-\hat{u}^{*}\right\|^{2}\right) .
\end{aligned}
$$


Multiplying (5.17) by a small positive constant $\kappa$, adding the resultant together with (5.90), taking $\kappa$ small enough yields, and then recalling (5.79), we obtain

$$
\begin{aligned}
& \frac{d}{d t}\left[\frac{1}{2}\|\bar{u}\|^{2}+\frac{\kappa \eta}{2}\|\nabla \bar{u}\|^{2}+\frac{1}{2 k^{2}}\|\nabla \bar{f}\|^{2}+\frac{\varepsilon}{2}\|\bar{f}\|^{2}\right] \\
& +\frac{\eta}{4}\|\nabla \bar{u}\|^{2}+\frac{1}{16}\left\|\bar{f}_{t}\right\|^{2}+\frac{\varepsilon}{k^{2}}\|\nabla \bar{f}\|^{2}+\frac{\kappa}{2}\left\|\bar{u}_{t}\right\|^{2} \\
\leq & C\left(\|\bar{f}\|^{2}+\left\|\hat{u}-\hat{u}^{*}\right\|^{2}+\left\|\hat{u}_{t}\right\|^{2}\right) \\
\leq & C\left(t^{-2 \sigma}+t^{-2(1+\gamma)}+t^{-2(2+\gamma)}\right) .
\end{aligned}
$$

Now set

$$
y(t)=\frac{1}{2}\|\bar{u}(t)\|^{2}+\frac{\kappa \eta}{2}\|\nabla \bar{u}(t)\|^{2}+\frac{1}{2 k^{2}}\|\nabla \bar{f}(t)\|^{2}+\frac{\varepsilon}{2}\|\bar{f}(t)\|^{2}
$$

and observe that (5.91) yields

$$
\frac{d}{d t} y(t)+C y(t) \leq C\left(t^{-2 \sigma}+t^{-2(1+\gamma)}+t^{-2(2+\gamma)}\right) \leq C t^{-2 \sigma}, \quad t \geq t_{0}^{*}
$$

Then the Gronwall inequality entails

$$
y(t) \leq C t^{-2 \sigma}, \quad t \geq t_{0}^{*},
$$

which finally implies (5.27). The proof of Theorem 5.1 is now complete.

6. Appendix. Here we want to discuss two meaningful cases in which it is possible to construct a finite-dimensional exponential attractor and, consequently, to deduce that the uniform attractor (cf. Section 4) has finite fractal dimension as well. The first case concerns a quasi-periodic $\tilde{u}$, and in the second one we will deal with a stabilizing $\tilde{u}$. The latter is closely related to Section 5. Before discussing these examples, we need to establish two further basic properties of the process $\left\{U_{\tilde{u}}(t, \tau), \tilde{u} \in H\left(\tilde{u}^{\#}\right)\right\}$, namely, the Hölder continuity with respect to time of the trajectories and the so-called smoothing property (see [12]). The former is given by

Lemma 6.1. Let $\tilde{u}^{\#} \in \mathcal{T}$. If $\left(f_{0}, u_{0}\right) \in \mathcal{H}$, then there exists $t_{0}>\tau$ such that, for any $t \geq t_{0}$ and each $s \in(0,1)$,

$$
\|f(t+s)-f(t)\|_{H^{1}}+\|u(t+s)-u(t)\| \leq C\left(\left\|\left(f_{0}, u_{0}\right)\right\|_{\mathcal{H}},\left\|\tilde{u}^{\#}\right\|_{\mathcal{T}}\right) \sqrt{s} .
$$

Proof. Recalling Lemma 4.1, it is easy to see that

$$
\begin{aligned}
& \|f(t+s)-f(t)\|_{H^{1}}^{2} \\
\leq & C\|f(t+s)-f(t)\|\|f(t+s)-f(t)\|_{H^{2}} \\
\leq & C\|f(t+s)-f(t)\|_{H^{2}} \int_{t}^{t+s}\left\|f_{t}(\tau)\right\| d \tau \\
\leq & C\left(\|f(t+s)\|_{H^{2}}+\|f(t)\|_{H^{2}}\right) \int_{t}^{t+s}\left\|\frac{1}{k^{2}} \Delta f-\left(f^{2}-1+u+\hat{u}\right) f\right\| d \tau \\
\leq & C\left(\left\|\left(f_{0}, u_{0}\right)\right\|_{\mathcal{H}},\|\tilde{u}\|_{\mathcal{T}}\right) s
\end{aligned}
$$


and

$$
\begin{aligned}
& \|u(t+s)-u(t)\|^{2} \\
\leq & \|u(t+s)-u(t)\|_{H^{1}} \int_{t}^{t+s}\left\|u_{t}\right\|_{\left(H^{1}\right)^{\prime}} d \tau \\
\leq & \left(\|u(t+s)\|_{H^{1}}+\|u(t)\|_{H^{1}}\right) \int_{t}^{t+s}\left\|f f_{t}+\eta \Delta u-\hat{u}_{t}\right\|_{\left(H^{1}\right)^{\prime}} d \tau \\
\leq & \left(\|u(t+s)\|_{H^{1}}+\|u(t)\|_{H^{1}}\right) \int_{t}^{t+s}\left(\left\|f_{t}\right\|\|f\|_{L^{\infty}}+\|\nabla u\|+\left\|\hat{u}_{t}\right\|\right) d \tau \\
\leq & C\left(\left\|\left(f_{0}, u_{0}\right)\right\|_{\mathcal{H}},\|\tilde{u}\|_{\mathcal{T}}\right)\left(s+s^{\frac{1}{2}}\right) .
\end{aligned}
$$

The proof is thus complete.

Let us now prove the smoothing property. First consider a uniform absorbing set $\mathcal{B}_{1}$ bounded in $H^{2}(\Omega) \times H_{0}^{1}(\Omega)$ given by Lemma 4.1 and let

$$
\left(f_{0 i}, u_{0 i}\right) \in \mathcal{B}_{1}, \quad i=1,2 .
$$

Moreover, suppose

$$
\tilde{u}^{\#} \in \mathcal{T} \cap C_{l o c}\left(\mathbb{R} ; H^{\frac{1}{2}}(\Gamma)\right)
$$

and take $\tilde{u}_{i} \in H\left(\tilde{u}^{\#}\right), i=1,2$. Then again set

$$
\begin{aligned}
f:=f_{1}-f_{2}, \quad u:=u_{1}-u_{2}, \quad \hat{u}:=\hat{u}_{1}-\hat{u}_{2}, \\
f_{0}:=f_{01}-f_{02}, \quad u_{0}:=u_{01}-u_{02}, \quad \tilde{u}:=\tilde{u}_{1}-\tilde{u}_{2} .
\end{aligned}
$$

We have

Lemma 6.2. Let (6.5) and (6.6) hold. Then, for any $T>\tau$, there is a positive constant $C(T)$ such that

$$
\begin{aligned}
& \|\Delta f(T)\|^{2}+\|\nabla u(T)\|^{2} \\
& \leq(T-\tau)^{-1} C(T)\left[\left\|u_{0}\right\|^{2}+\left\|f_{0}\right\|_{H^{1}}^{2}+\int_{\tau}^{T}\left(\|\tilde{u}(t)\|_{H^{\frac{1}{2}}(\Gamma)}^{2}+\left\|\tilde{u}_{t}(t)\right\|_{H^{-\frac{1}{2}}(\Gamma)}^{2}\right) d t\right],
\end{aligned}
$$

where $C(T)$ also depends on $\left\|f_{0 i}\right\|_{H^{2}},\left\|u_{0 i}\right\|_{H^{1}},\left\|\tilde{u}_{i}\right\|_{C\left([\tau, T], H^{\frac{1}{2}}(\Gamma)\right)}$, and $\tau$.

Proof.

$$
\begin{gathered}
f_{t}=\frac{1}{k^{2}} \Delta f-\left(u_{1} f_{1}-u_{2} f_{2}\right)-\left(\hat{u}_{1} f_{1}-\hat{u}_{2} f_{2}\right)-\left(\phi\left(f_{1}\right)-\phi\left(f_{2}\right)\right), \\
u_{t}-f_{1} f_{1 t}+f_{2} f_{2 t}=\eta \Delta u-\hat{u}_{t},
\end{gathered}
$$

where we have set $\phi(\xi)=\xi^{3}-\xi$. 
Multiplying (6.10) by $-\Delta f_{t}$ and integrating on $\Omega$, we get

$$
\begin{aligned}
& \frac{1}{2 k^{2}} \frac{d}{d t}\|\Delta f\|^{2}+\left\|\nabla f_{t}\right\|^{2} \\
& =\int_{\Omega}\left(u_{1} f_{1}-u_{2} f_{2}\right) \Delta f_{t} d x+\int_{\Omega}\left(\hat{u}_{1} f_{1}-\hat{u}_{2} f_{2}\right) \Delta f_{t} d x \\
& \quad+\int_{\Omega}\left(\phi\left(f_{1}\right)-\phi\left(f_{2}\right)\right) \Delta f_{t} d x \\
& \int_{\Omega}\left(\phi\left(f_{1}\right)-\phi\left(f_{2}\right)\right) \Delta f_{t} d x \\
& \quad=-\int_{\Omega}\left(\phi^{\prime}\left(f_{1}\right) \nabla f_{1}-\phi^{\prime}\left(f_{2}\right) \nabla f_{2}\right) \cdot \nabla f_{t} d x \\
& \quad=-\int_{\Omega}\left(\phi^{\prime}\left(f_{1}\right)-\phi^{\prime}\left(f_{2}\right)\right) \nabla f_{1} \cdot \nabla f_{t} d x-\int_{\Omega} \phi^{\prime}\left(f_{2}\right) \nabla f \cdot \nabla f_{t} d x \\
& \quad:=I_{4}+I_{5} .
\end{aligned}
$$

Observe now that

$$
\begin{gathered}
\left|I_{4}\right| \leq \epsilon\left\|\nabla f_{t}\right\|^{2}+C_{\epsilon}\left\|\left(\phi^{\prime}\left(f_{1}\right)-\phi^{\prime}\left(f_{2}\right)\right) \nabla f_{1}\right\|^{2} \\
\leq \epsilon\left\|\nabla f_{t}\right\|^{2}+C_{\epsilon}\|f\|_{L^{4}}^{2}\left\|\nabla f_{1}\right\|_{L^{4}}^{2} \\
\leq \epsilon\left\|\nabla f_{t}\right\|^{2}+C_{\epsilon}\|f\|_{H^{1}}^{2} \\
\quad\left|I_{5}\right| \leq \epsilon\left\|\nabla f_{t}\right\|^{2}+C_{\epsilon}\|\nabla f\|^{2} .
\end{gathered}
$$

On the other hand, we have

$$
\int_{\Omega}\left(u_{1} f_{1}-u_{2} f_{2}\right) \Delta f_{t} d x=\int_{\Omega} u f_{1} \Delta f_{t} d x+\int_{\Omega} u_{2} f \Delta f_{t} d x:=I_{6}+I_{7},
$$

with

$$
\begin{aligned}
\left|I_{6}\right| & \leq\left|\int_{\Omega} f_{1} \nabla u \cdot \nabla f_{t} d x\right|+\left|\int_{\Omega} u \nabla f_{1} \cdot \nabla f_{t} d x\right| \\
& \leq \epsilon\left\|\nabla f_{t}\right\|^{2}+C_{\epsilon}\left(\|\nabla u\|^{2}\left\|f_{1}\right\|_{L^{\infty}}^{2}+\|u\|_{L^{4}}^{2}\left\|\nabla f_{1}\right\|_{L^{4}}^{2}\right) \\
& \leq \epsilon\left\|\nabla f_{t}\right\|^{2}+C_{\epsilon}\|\nabla u\|^{2}, \\
\left|I_{7}\right| & \leq\left|\int_{\Omega} f \nabla u_{2} \cdot \nabla f_{t} d x\right|+\left|\int_{\Omega} u_{2} \nabla f \cdot \nabla f_{t} d x\right| \\
& \leq \epsilon\left\|\nabla f_{t}\right\|^{2}+C_{\epsilon}\left(\left\|\nabla u_{2}\right\|^{2}\|f\|_{L^{\infty}}^{2}+\left\|u_{2}\right\|_{L^{4}}^{2}\|\nabla f\|_{L^{4}}^{2}\right) \\
& \leq \epsilon\left\|\nabla f_{t}\right\|^{2}+C_{\epsilon}\|f\|_{H^{2}}^{2} .
\end{aligned}
$$

Similarly, we find

$$
\left|\int_{\Omega}\left(\hat{u}_{1} f_{1}-\hat{u}_{2} f_{2}\right) \Delta f_{t} d x\right| \leq \epsilon\left\|\nabla f_{t}\right\|^{2}+C_{\epsilon}\|\nabla \hat{u}\|^{2}+C_{\epsilon}\left\|\nabla \hat{u}_{2}\right\|^{2}\|f\|_{H^{2}}^{2} .
$$

Multiplying (6.11) by $-\Delta u$, integrating on $\Omega$, we obtain

$$
\frac{1}{2} \frac{d}{d t}\|\nabla u\|^{2}+\eta\|\Delta u\|^{2}=\int_{\Omega}\left(-f_{1} f_{1 t}+f_{2} f_{2 t}\right) \Delta u d x+\int_{\Omega} \hat{u}_{t} \Delta u d x
$$


Then there holds

Moreover, setting

$$
\left|\int_{\Omega} \hat{u}_{t} \Delta u d x\right| \leq \epsilon \eta\|\Delta u\|^{2}+C_{\epsilon}\left\|\hat{u}_{t}\right\|^{2} .
$$

$$
\int_{\Omega}\left(-f_{1} f_{1 t}+f_{2} f_{2 t}\right) \Delta u d x=-\int_{\Omega} f f_{1 t} \Delta u d x-\int_{\Omega} f_{2} f_{t} \Delta u d x:=I_{8}+I_{9},
$$

we observe that

$$
\left|I_{8}\right| \leq \epsilon \eta\|\Delta u\|^{2}+C_{\epsilon}\|f\|_{L^{\infty}}^{2}\left\|f_{1 t}\right\|^{2} \leq \epsilon \eta\|\Delta u\|^{2}+C_{\epsilon}\|f\|_{H^{2}}^{2}\left\|f_{1 t}\right\|^{2},
$$

where

$$
\left\|f_{1 t}\right\| \leq C\left(\left\|\Delta f_{1}\right\|^{2}+\left\|f_{1}\right\|_{H^{2}}^{3}+\left\|f_{1}\right\|_{H^{2}}+\left\|f_{1}\right\|_{H^{2}}\left\|u_{1}\right\|+\left\|f_{1}\right\|_{H^{2}}\left\|\hat{u}_{1}\right\|\right) \leq C\left(1+\left\|\hat{u}_{1}\right\|\right),
$$

and

$$
\left|I_{9}\right| \leq \epsilon \eta\|\Delta u\|^{2}+C_{\epsilon}\left\|f_{2}\right\|_{L^{\infty}}^{2}\left\|f_{t}\right\|^{2} \leq \epsilon \eta\|\Delta u\|^{2}+C_{\epsilon}\left\|f_{t}\right\|^{2} .
$$

Summing up, we infer

$$
\begin{aligned}
& \frac{d}{d t}\left(\frac{1}{2 k^{2}}\|\Delta f\|^{2}+\frac{1}{2}\|\nabla u\|^{2}\right)+\frac{1}{2}\left\|\nabla f_{t}\right\|^{2}+\frac{\eta}{2}\|\Delta u\|^{2} \\
\leq & C\left(1+\left\|\hat{u}_{1}\right\|^{2}+\left\|\nabla \hat{u}_{2}\right\|^{2}\right)\|f\|_{H^{2}}^{2}+C\|\nabla u\|^{2}+C\left\|f_{t}\right\|^{2} .
\end{aligned}
$$

Recalling (6.6), it follows from (3.31)-(3.36) that

$$
\begin{aligned}
& \int_{\tau}^{T}\left(\|\nabla u(t)\|^{2}+\|f(t)\|_{H^{1}}^{2}+\left\|f_{t}(t)\right\|^{2}\right) d t \\
\leq & 2 e^{\beta(T)}\left(\left\|u_{0}\right\|^{2}+\left\|f_{0}\right\|_{H^{1}}^{2}\right)+2 C e^{\beta(T)} \int_{\tau}^{T}\left(\|\hat{u}(t)\|_{H^{1}}^{2}+\left\|\hat{u}_{t}(t)\right\|^{2}\right) d t,
\end{aligned}
$$

which also implies

$$
\int_{\tau}^{T}\|\Delta f(t)\|^{2} d t \leq C(T)\left[\left\|u_{0}\right\|^{2}+\left\|f_{0}\right\|_{H^{1}}^{2}+\int_{\tau}^{T}\left(\|\hat{u}(t)\|_{H^{1}}^{2}+\left\|\hat{u}_{t}(t)\right\|^{2}\right) d t\right] .
$$

We now multiply (6.26) by $t-\tau$ and integrate from $\tau$ to $T$,

$$
\begin{aligned}
& (T-\tau)\left(\|\Delta f(T)\|^{2}+\|\nabla u(T)\|^{2}\right) \\
\leq & C(T)\left[\left\|u_{0}\right\|^{2}+\left\|f_{0}\right\|_{H^{1}}^{2}+\int_{\tau}^{T}\left(\|\hat{u}(t)\|_{H^{1}}^{2}+\left\|\hat{u}_{t}(t)\right\|^{2}\right) d t\right],
\end{aligned}
$$

which yields (6.9).

6.1. Quasi-periodic boundary data. Let us consider a function $G\left(z_{1}, \ldots, z_{k}\right): \mathbb{T}^{k} \rightarrow$ $H^{\frac{1}{2}}(\Gamma)$ which is $2 \pi$-periodic with respect to each argument $z_{i}(i=1, \ldots, k)$ and such that

$$
G \in C^{1}\left(\mathbb{T}^{k} ; H^{\frac{1}{2}}(\Gamma)\right) \cap C^{2}\left(\mathbb{T}^{k} ; H^{-\frac{1}{2}}(\Gamma)\right) .
$$

Then we define

$$
\tilde{u}^{\#}(t)=G(\boldsymbol{\alpha} t), \quad \forall t \in \mathbb{R},
$$

with $\boldsymbol{\alpha} \in \mathbb{R}^{k}$ being a vector with rationally independent frequencies (see, e.g., [8, 13]). Consequently, we introduce the symbol space

$$
\Sigma=\left\{\tilde{u}\left(\alpha_{1} t+\sigma_{1}, \ldots, \alpha_{k} t+\sigma_{k}\right), \boldsymbol{\sigma}=\left(\sigma_{1}, \ldots, \sigma_{k}\right) \in \mathbb{T}^{k}\right\},
$$


which can be topologically identified with the torus $\mathbb{T}^{k}$, and we observe that

$$
\left\|\left(\tilde{u}_{1}-\tilde{u}_{2}\right)(t)\right\|_{H^{\frac{1}{2}(\Gamma)}}^{2}+\left\|\left(\tilde{u}_{1 t}-\tilde{u}_{2 t}\right)(t)\right\|_{H^{-\frac{1}{2}(\Gamma)}}^{2} \leq C_{G}\left|\boldsymbol{\sigma}_{1}-\boldsymbol{\sigma}_{2}\right|^{2},
$$

for all $t \in \mathbb{R}$, where $\tilde{u}_{i}(t)=\tilde{u}\left(\boldsymbol{\alpha} t+\boldsymbol{\sigma}_{i}\right), i=1,2$.

Then, for each $\sigma \in \mathbb{T}^{k}$, we consider the process

$$
U_{F_{h}}(t, \tau)=U_{\boldsymbol{\sigma}}(t+h, \tau+h), \quad \forall h \geq 0, t \geq \tau, \tau \in \mathbb{R},
$$

where

$$
F_{h} \boldsymbol{\sigma}:=(\boldsymbol{\alpha} h+\boldsymbol{\sigma}) \bmod (2 \pi)^{k} .
$$

This process can be interpreted as an extended semigroup (see Section 4)

$$
S(t): \mathcal{H} \times \mathbb{T}^{k} \rightarrow \mathcal{H} \times \mathbb{T}^{k},
$$

by setting

$$
S(t)\left(f_{0}, u_{0}, \boldsymbol{\sigma}\right):=\left(U_{\boldsymbol{\sigma}}(t, 0)\left(f_{0}, u_{0}\right), F_{t} \boldsymbol{\sigma}\right) .
$$

Because of Lemma 4.1. we know that $S(t)$ has a compact absorbing set bounded in $H^{2}(\Omega) \times H^{1}(\Omega) \times \mathbb{T}^{k}$. Therefore, $S(t)$ has the global attractor $\mathbb{A}$.

Thanks to (6.33) and Lemmas 6.1 and 6.2, we can argue as in [13, (see also [16, 36]) and prove the following

Theorem 6.1. Let $G$ satisfy (6.30) and let $\tilde{u}^{\#}$ be given by (6.31). Then the extended semigroup $S(t)$ has an exponential attractor $\mathbb{M}$ of finite fractal dimension. Moreover, we have

(i) $\Pi_{1} \mathbb{M}=\mathcal{M}$ is a uniform exponential attractor for the family $\left\{U_{\tilde{u}}(t, \tau), \tilde{u} \in \Sigma\right\}$;

(ii) $\Pi_{2} \mathbb{M}=\Sigma$;

(iii) the global attractor $\mathcal{A}$ of the family $\left\{U_{\tilde{u}}(t, \tau), \tilde{u} \in \Sigma\right\}$ has finite fractal dimension.

REMARK 6.1. $\mathcal{M}$ is bounded in $H^{2}(\Omega) \times H_{0}^{1}(\Omega)$.

6.2. Stabilizing boundary data. Let us consider

$$
\tilde{u} \in H^{1}\left(\mathbb{R} ; H^{\frac{1}{2}}(\Gamma)\right) .
$$

Suppose that there are two functions $\tilde{u}^{*}, \tilde{u}_{*} \in H^{\frac{1}{2}}(\Gamma)$ such that, for all $t>0$,

$$
\int_{t}^{t+1}\left(\left\|\tilde{u}(y)-\tilde{u}^{*}\right\|_{H^{\frac{1}{2}}(\Gamma)}^{2}+\left\|\tilde{u}_{t}(y)\right\|_{H^{-\frac{1}{2}}(\Gamma)}^{2}\right) d y \leq C t^{-\alpha}
$$

and, for all $t<0$,

$$
\int_{t}^{t+1}\left(\left\|\tilde{u}(y)-\tilde{u}_{*}\right\|_{H^{\frac{1}{2}}(\Gamma)}^{2}+\left\|\tilde{u}_{t}(y)\right\|_{H^{-\frac{1}{2}}(\Gamma)}^{2}\right) d y \leq C|t|^{-\alpha},
$$

for some $C>0$ and some $\alpha>0$. Then we have

$$
H(\tilde{u})=\left\{\tilde{u}^{*}\right\} \cap\left\{\tilde{u}_{*}\right\} \cap\{\tilde{u}(\cdot+h) \mid h \in \mathbb{R}\},
$$

and this hull has finite fractal dimension (see [13, Ex. 7.4]). Moreover, due to (6.38), there exists a positive constant $c$ such that

$$
\int_{t}^{t+1}\|\tilde{u}(y)-\tilde{u}(y+h)\|_{H^{\frac{1}{2}}(\Gamma)}^{2} d y \leq c h, \quad \forall h \in \mathbb{R} .
$$

We now have all the ingredients to argue as in the previous case. Thus we can state 
TheOREm 6.2. Let (6.38)-(6.40) hold. Then the extended semigroup $S(t)$ acting on $\mathcal{H} \times H(\tilde{u})$ has an exponential attractor $\mathbb{M}$ of finite fractal dimension. Moreover, properties (i)-(iii) of Theorem 6.1 are still valid.

REMARK 6.2. For a more general notion of an exponential attractor for a nonautonomous system, which does not necessarily have finite fractal dimension, we refer the reader to [13, 14.

Acknowledgments. Part of this paper was written during the first author's visit to the Institute of Mathematics in Fudan University, whose hospitality is gratefully acknowledged. The first author has been partially supported by the Italian PRIN Research Project 2006 Problemi a frontiera libera, transizioni di fase e modelli di isteresi. The third author was supported by the NSF of China under grant No. 10631020 and by the Chinese ministry of education under grant No. 20050246002.

\section{REFERENCES}

[1] S. Aizicovici, E. Feireisl, F. Issard-Roch, Long time convergence of solutions to a phase-field system, Math. Methods Appl. Sci. 24 (2001), 277-287. MR1818896 (2002b:35081)

[2] P.W. Bates, S. Zheng, Inertial manifolds and inertial sets for the phase-field equations, J. Dynamics Differential Equations 4 (1992), 375-397. MR1160925 (93h:35187)

[3] V. Berti, M. Fabrizio, A non-isothermal Ginzburg-Landau model in superconductivity: existence, uniqueness and asymptotic behavior, Nonlinear Anal. 66 (2007), 2565-2578. MR2312606 (2008a:82098)

[4] D. Brochet, X. Chen, D. Hilhorst, Finite dimensional exponential attractor for the phase-field model, Appl. Anal. 49 (1993), 197-212. MR.1289743 (95g:35097)

[5] D. Brochet, D. Hilhorst, Universal attractor and inertial sets for the phase-field model, Appl. Math. Lett. 4 (1991), 59-62. MR.1136614 (92g:35102)

[6] M. Brokate, J. Sprekels, Hysteresis and Phase Transitions, Appl. Math. Sci. 121, Springer, New York, 1996. MR 1411908 (97g:35127)

[7] G. Caginalp, An analysis of a phase field model of a free boundary, Arch. Ration. Mech. Anal. 92 (1986), 205-245. MR816623 (87c:80011)

[8] V.V. Chepyzhov, M.I. Vishik, Attractors for Equations of Mathematical Physics, Amer. Math. Soc. Colloq. Publ. 49, AMS, Providence, RI, 2002. MR 1868930 (2003f:37001c)

[9] L. Cherfils, A. Miranville, Some remarks on the asymptotic behavior of the Caginalp system with singular potentials, Adv. Math. Sci. Appl. 16 (2007), 107-129. MR2337372

[10] L. Cherfils, A. Miranville, On the Caginalp system with dynamic boundary conditions and singular potentials, Appl. Math., to appear.

[11] R. Chill, M.A. Jendoubi, Convergence to steady states in asymptotically autonomous semilinear evolution equations, Nonlinear Anal. 53 (2003), 1017-1039. MR.1978032 (2004d:34103)

[12] M. Efendiev, A. Miranville, S. Zelik, Exponential attractors for a nonlinear reaction-diffusion system in $\mathbb{R}^{3}$, C. R. Math. Acad. Sci. Paris 330 (2000), 713-718. MR.1763916 (2001c:35039)

[13] M. Efendiev, A. Miranville, S. Zelik, Infinite dimensional exponential attractors for a nonautonomous reaction-diffusion system, Math. Nachr. 248/249 (2003), 72-96. MR1950716 (2003i:37082)

[14] M. Efendiev, S. Zelik, A. Miranville, Exponential attractors and finite-dimensional reduction for non-autonomous dynamical systems. Proc. Roy. Soc. Edinburgh Sect. A 135 (2005), 703-730. MR2173336 (2007a:37098)

[15] C.M. Elliott, S. Zheng, Global existence and stability of solutions to the phase-field equations, in "Free boundary problems", Internat. Ser. Numer. Math. 95, 46-58, Birkhäuser Verlag, Basel, 1990. MR 1111021 (92g:35214)

[16] P. Fabrie, A. Miranville, Exponential attractors for nonautonomous first-order evolution equations, Discrete Contin. Dynam. Syst. 4 (1998), 225-240. MR.1617294 (99c:34132) 
[17] M.Fabrizio, Ginzburg-Landau equations and first and second order phase transitions, Internat. J. Engrg. Sci. 44 (2006), 529-539. MR2234088 (2007f:82118)

[18] G.J. Fix, Phase field models for free boundary problems, in "Free boundary problems: theory and applications, Vol. II"(A. Fasano and M. Primicerio, eds.), Pitman Res. Notes Math. Ser. 79, 580-589, Longman, London, 1983.

[19] C.G. Gal, M. Grasselli, The nonisothermal Allen-Cahn equation with dynamic boundary conditions, Discrete Contin. Dynam. Syst., 22 (2008), 1009-1040.

[20] S. Gatti, A. Miranville, Asymptotic behavior of a phase-field system with dynamic boundary conditions, in "Differential Equations Inverse and Direct Problems"(A. Favini and A. Lorenzi, eds.), Ser. Lect. Notes Pure Appl. Math. 251, 149-170, Chapman \& Hall/CRC, Boca Raton, 2006. MR 2275977 (2007g:35087)

[21] C. Giorgi, M. Grasselli, V. Pata, Uniform attractors for a phase-field model with memory and quadratic nonlinearity, Indiana Univ. Math. J. 48 (1999), 1395-1445. MR.1757078 (2001h:37160)

[22] M. Grasselli, V. Pata, Asymptotic behavior of a parabolic-hyperbolic system, Commun. Pure Appl. Anal. 3 (2004), 849-881. MR2106302(2005h:35150)

[23] M. Grasselli, H. Petzeltová, G. Schimperna, Long time behavior of solutions to the Caginalp system with singular potential, Z. Anal. Anwendungen 25 (2006), 51-72. MR2216881(2007b:35159)

[24] M. Grasselli, H. Petzeltová, G. Schimperna, Convergence to stationary solutions for a parabolichyperbolic phase-field system, Commun. Pure Appl. Anal. 5 (2006), 827-838. MR2246010 (2007g:35088)

[25] J.K. Hale, Asymptotic behavior of dissipative systems, Math. Surveys Monogr. 25, Amer. Math. Soc., Providence, 1988. MR941371 (89g:58059)

[26] A. Haraux, Systèmes dynamiques dissipatifs et applications, Recherches en Mathématiques Appliquées 17, Masson, Paris, 1991. MR:1084372 (92b:35002)

[27] A. Haraux, M.A. Jendoubi, Convergence of bounded weak solutions of the wave equation with dissipation and analytic nonlinearity, Calc. Var. Partial Differential Equations 9 (1999), 95-124. MR:1714129 (2000h:35110)

[28] S.-Z. Huang, P. Takáč, Convergence in gradient-like systems which are asymptotically autonomous and analytic, Nonlinear Anal. 46 (2001), 675-698. MR1857152 (2002f:35125)

[29] M.A. Jendoubi, A simple unified approach to some convergence theorem of L. Simon, J. Funct. Anal. 153 (1998), 187-202. MR.1609269 (99c:35101)

[30] A. Jiménez-Casas, A. Rodríguez-Bernal, Asymptotic behaviour for a phase field model in higher order Sobolev spaces, Rev. Mat. Complut. 15 (2002), 213-248. MR.1915223 (2003f:35148)

[31] V.K. Kalantarov, On the minimal global attractor of a system of phase field equations (Russian), Zap. Nauchn. Sem. Leningrad. Otdel. Mat. Inst. Steklov. (LOMI) 188 (1991), Kraev. Zadachi Mat. Fiz. i Smezh. Voprosy Teor. Funktsii. 22, 70-86, 186 [translation in J. Math. Sci. 70 (1994), no. 3, 1767-1777]. MR1111469 (93f:35121)

[32] O.V. Kapustyan, An attractor of a semiflow generated by a system of phase-field equations without uniqueness of the solution (Ukrainian), Ukraïn. Mat. Zh. 51 (1999), 1006-1009 [Translation in Ukrainian Math. J. 51 (1999), no. 7, 1135-1139 (2000)]. MR.1727706 (2001b:37113)

[33] Ph. Laurençot, Long-time behaviour for a model of phase-field type, Proc. Roy. Soc. Edinburgh Sect. A 126 (1996), 167-185. MR 1378839(97a:35115)

[34] S. Ma, C. Zhong, The attractor for weakly damped non-autonomous hyperbolic equations with a new class of external forces, Discrete Contin. Dynam. Syst. 18 (2007), 53-70. MR 2276486

[35] G.B. McFadden, Phase-field models of solidification, Contemp. Math. 306 (2002), 107-145. MR $1940624(2003 \mathrm{k}: 80004)$

[36] A. Miranville, Exponential attrators for nonautonomous evolution equations, Appl. Math. Lett. 11 (1998), 19-22. MR1609661

[37] N. Sato, T. Aiki, Phase field equations with constraints under nonlinear dynamic boundary conditions, Commun. Appl. Anal. 5 (2001), 215-234. MR1844192 (2002f:35131)

[38] G. Schimperna, Abstract approach to evolution equations of phase field type and applications, J. Differential Equations 164 (2000), 395-430. MR.1765571 (2001h:35110)

[39] H. Wu, M. Grasselli, S. Zheng, Convergence to equilibrium for a parabolic-hyperbolic phase-field system with Neumann boundary conditions, Math. Models Methods Appl. Sci. 17 (2007) 125-153. MR 2290411.

[40] Z. Zhang, Asymptotic behavior of solutions to the phase-field equations with Neumann boundary conditions, Commun. Pure Appl. Anal. 4 (2005), 683-693. MR2167193 (2006i:35142) 
[41] S. Zheng, Nonlinear Evolution Equations, Chapman \& Hall/CRC Monogr. Surv. Pure Appl. Math. 133, Chapman \& Hall/CRC, Boca Raton, Florida, 2004. MR2088362 (2006a:35001) 